NBER WORKING PAPER SERIES

\title{
AN ANALYTIC FRAMEWORK FOR INTERPRETING INVESTMENT REGRESSIONS IN THE PRESENCE OF FINANCIAL CONSTRAINTS
}

\author{
Andrew B. Abel \\ Stavros Panageas \\ Working Paper 26898 \\ http://www.nber.org/papers/w26898 \\ NATIONAL BUREAU OF ECONOMIC RESEARCH \\ 1050 Massachusetts Avenue \\ Cambridge, MA 02138 \\ March 2020
}

Previous versions circulated as "The Impact of Financial Constraints on Investment, Dividends, and q." We thank participants in seminars at the Federal Reserve Banks of Atlanta and St. Louis and the Penn Macro Lunch Group for helpful comments. The views expressed herein are those of the authors and do not necessarily reflect the views of the National Bureau of Economic Research.

NBER working papers are circulated for discussion and comment purposes. They have not been peer-reviewed or been subject to the review by the NBER Board of Directors that accompanies official NBER publications.

(C) 2020 by Andrew B. Abel and Stavros Panageas. All rights reserved. Short sections of text, not to exceed two paragraphs, may be quoted without explicit permission provided that full credit, including (C) notice, is given to the source. 
An Analytic Framework For Interpreting Investment Regressions In The Presence Of Financial Constraints

Andrew B. Abel and Stavros Panageas

NBER Working Paper No. 26898

March 2020

JEL No. E22,G33,G35

\begin{abstract}
A financial constraint that prevents access to external funds induces non-classical measurement error in average $\mathrm{q}$ as a proxy for unobservable marginal $\mathrm{q}$. Unlike classical measurement error, this measurement error biases upward the coefficient on average $q$ in a univariate regression of investment on average $q$. In a multiple regression of investment on average $q$ and cash flow, the coefficient on cash flow is positive. The positive cash-flow coefficient indicates the presence of a financial constraint, but it does not indicate a shortage of liquidity to fund current investment. In addition, the coefficient on average $\mathrm{q}$ is biased downward.
\end{abstract}

\author{
Andrew B. Abel \\ The Wharton School \\ University of Pennsylvania \\ 2315 Steinberg Hall - Dietrich Hall \\ Philadelphia, PA 19104-6367 \\ and NBER \\ abel@wharton.upenn.edu \\ Stavros Panageas \\ Anderson School of Management \\ University of California, Los Angeles \\ 110 Westwood Plaza \\ Los Angeles, CA 90095-1481 \\ and NBER \\ stavros.panageas@anderson.ucla.edu
}




\section{Introduction}

Financial constraints can affect a firm's optimal capital investment, even when the constraints are not binding. They can also affect the relationship between investment and average $q$, without changing the relationship between investment and suitably-defined marginal $q$. We develop a simple dynamic stochastic model of a firm that faces an explicit financial constraint and we derive the optimal capital investment and payout decisions of the firm. We also derive the value of the firm, which allows us to derive expressions for average $q$ and marginal $q$. We show that even when the financial constraint is not binding, it reduces the value of the firm, thereby reducing average $q$, marginal $q$, and investment. More interesting is our finding that the financial constraint derives a wedge between marginal $q$ and its commonlyused empirical proxy, average $q$, even if average $q$ and marginal $q$ would be identically equal to each other in an otherwise-identical model without financial constraints. This wedge has important implications for regressions of the investment-capital ratio on average $q$, with or without cash flow as an additional regressor. Importantly, we demonstrate that the estimated coefficient on cash flow in investment regressions conflates the impacts of financial constraints and the impact of the expected present value of future profitability.

By design, the non-financial aspects of the firm's decision problem are simple in order to allow explicit solutions for various variables of interest. The firm's operating profit at any point in time is the product of an exogenous profitability parameter and the capital stock. The exogenous profitability parameter alternates stochastically in continuous time between a positive value and a negative value, according to a Markov regime-switching process. The firm can change its capital stock by purchasing or selling capital at a price normalized to one and incurring an adjustment cost. The adjustment cost function is linearly homogeneous in the rate of investment and the level of the capital stock and is convex in the rate of investment. Therefore, the non-financial environment satisfies the Hayashi conditions, ${ }^{1}$ so average $q$ would equal marginal $q$ in the absence of financial constraints. Consistent with much of the literature, the adjustment cost function is quadratic in the investment-capital ratio, so the optimal investment-capital ratio is linear in marginal $q$.

To obtain closed-form expressions for various aspects of the firm's valuation and optimal behavior, we consider the limiting case in which the adjustment cost scale factor becomes arbitrarily large. Rather than leading to very large adjustment costs when the firm im-

\footnotetext{
${ }^{1}$ The proportionality of profit to the capital stock and the linear homogeneity of the cost of investment together satisfy the Hayashi conditions.
} 
plements its optimal investment policy, the total adjustment cost becomes negligible as the adjustment cost scale factor becomes arbitrarily large. Nevertheless, as we show, the realized marginal adjustment cost does not vanish, so optimal investment is given by the standard equalization of marginal $q$ and one plus the marginal adjustment cost.

We introduce financial constraints in a very stark form. We assume that once a firm acquires and installs its initial capital stock, it cannot raise any external funds. The firm cannot issue debt or equity, and it cannot pay negative dividends. When the favorable regime prevails, the firm reaps positive profits from operations, which it can use to pay dividends, undertake investment, or accumulate cash on hand. However, when the unfavorable regime prevails, the firm has negative profits from operations. In order to be able to pay costs in excess of revenues, the firm must use some of its accumulated cash on hand. ${ }^{2}$ If the firm runs out of cash while profits are negative, it must terminate immediately and permanently, thereby losing its claim on future cash flows from operations. If the anticipated foregone cash flows are sufficiently large, the firm has an incentive to accumulate cash on hand in the favorable regime in order to allow it to survive for at least a while when the next unfavorable regime arrives. Shareholders balance this incentive to accumulate cash on hand against their desire to receive dividends sooner rather than later.

In the presence of the financial constraints we analyze, cash on hand becomes a second asset held by the firm in addition to its physical capital stock, so the firm's overall optimization problem has three state variables (profitability, capital, and cash on hand). The firm manages its two assets, cash and capital, through its payout and investment decisions, respectively. The payout decision determines how much of its cash flow net of investment the firm pays out as dividends and how much it retains within the firm. Importantly, the addition of cash on hand as a state variable destroys the equality of average $q$ and marginal $q$ despite the fact that the non-financial aspects of the firm satisfy the Hayashi conditions. Nevertheless, the value function of the firm is linearly homogeneous in capital and cash on hand, and we exploit this homogeneity to obtain closed-form solutions for the value of the firm as well as for optimal investment and dividends. ${ }^{3}$

With the quadratic specification of adjustment costs, the optimal investment-capital

\footnotetext{
${ }^{2}$ The adjustment function is scaled so that any proceeds from selling capital, net of adjustment costs, will not be sufficient to pay the excess of operating costs over revenues.

${ }^{3}$ When the salvage value in the event of termination is zero, we can express the value of the firm per unit of capital as a closed-form function of the primitive parameters of the firm's problem. When the salvage value is positive, such a closed-form expression is not available, but we can express the value of the firm per unit of capital as a closed-form function of the primitive parameters of the firm's problem and $x^{*}$, the optimal target cash-to-capital ratio.
} 
ratio is a linear function of suitably-defined marginal $q$, which is unobservable. Typically, empirical analyses relate the investment-capital ratio to average $q$, adjusted for holdings of liquid assets. As a prelude to analyzing the relationship between the optimal investmentcapital ratio and average $q$, we first analyze the relationship between marginal $q$ and average $q$. In particular, we examine the coefficient on average $q$ in a univariate regression of marginal $q$ on average $q$. If average $q$ were simply marginal $q$ plus classical measurement error, the coefficient on average $q$ would be smaller than one. However, the error in average $q$ as a measure of marginal $q$ is not classical, and we show that the coefficient on average $q$ is likely to be greater than one. Remarkably, this effect tends to increase the apparent response of the optimal investment-capital ratio to average $q$, contrary to the conventional argument based on classical measurement error. As a result, the presence of financial constraints is unlikely to be the source of the small regression coefficients obtained in empirical investment-average $q$ relations, since financial constraints would induce the opposite bias.

Our analysis then explores the role of cash flow in regressions of the investment-capital ratio on both average $q$ and cash flow. Consistent with typical empirical findings, our analysis demonstrates that the introduction of cash flow as an additional regressor in investment regressions reduces the OLS coefficient on average $q$. Also consistent with typical empirical findings, the coefficient on cash flow is positive. The conventional interpretation is that the positive coefficient on cash flow reflects a shortage of cash on hand relative to desired investment expenditures. Our analysis points to a different interpretation. We demonstrate analytically that marginal $q$ is the sum of average $q$ and a term that reflects the financial constraint. The term reflecting the financial constraint is the product of an increasing function of the shadow value of cash on hand (minus one) and the value of the firm per unit of capital. The shadow value of cash on hand (minus one) is positive if and only if the financial constraint is binding. The cash flow coefficient in a regression of the investment-capital ratio on average $q$ and cash flow captures the effect of the financial constraint. However, the interpretation is not that the cash flow coefficient reflects a shortage of cash on hand to undertake desired investment. Moreover, the estimated coefficient on cash flow also contains a component reflecting a fraction of the impact of expected future profitability on investment, effectively taking some of this impact away from the estimated coefficient on average $q$.

We show that there is one situation in which average $q$ and marginal $q$ are equal in the presence of financial constraints. When the financial constraint is not binding, that is when dividends are positive, marginal $q$ and average $q$ are equal. In a sample of observations 
in which dividends are always positive, investment regressions have a coefficient on average $q$ that equals the inverse of the adjustment cost scale parameter and a coefficient on cash flow that equals zero. This finding is consistent with Fazzari et al. (1988), hereafter FHP, who argue that regressions of the investment-capital ratio on average $q$ would perform best when the sample is restricted to observations for which dividends are positive. However, this is not to say that financial constraints don't impact the firm's investment when it is paying dividends, since marginal $q$, and hence investment, is reduced by the presence of a contemporaneously nonbinding financial constraint that will bind in the future at some unknown time.

Section 1.1 reviews the literature on investment and $q$, including the impact of financial constraints on the relationship among investment, $q$, and cash flow. Section 2 presents the physical environment of the firm, which comprises profit from operations, capital accumulation, and costs of adjusting the capital stock. Financial constraints are introduced in Section 3. To serve as a baseline for assessing the impact of financial constraints, Section 3.1 analyzes the valuation of the firm in the absence of financial constraints. The valuation of the firm and optimal investment in the presence of these constraints are analyzed in Section 3.2. Financial constraints drive a wedge between average $q$ and marginal $q$, even under the Hayashi conditions. Section 4 analyzes this wedge and its implications for regressions of the investment-capital ratio on average $q$, with and without cash flow as an additional regressor. Financial constraints introduce the possibility that the firm will be forced to terminate at some point, but shareholders may receive some salvage value upon termination. Section 5 analyzes the impact of the salvage value on the firm's valuation and optimal investment. Concluding remarks are in Section 6.

\subsection{Literature Review}

The notion that a firm's optimal rate of investment is linked to its valuation originated in Keynes (1936). Brainard and Tobin (1968) gave empirical content to this link by defining a ratio that Tobin (1969) called " $q$," and the subsequent literature has dubbed Tobin's $q$. The numerator of this ratio is the market value of a firm and the denominator of this ratio is the replacement cost of the firm's capital stock. An appeal of Tobin's $q$ is that it relies on financial markets to evaluate the firm's current and future prospects, thereby relieving a researcher from having to perform the appropriate calculation of the expected present value of future marginal products of capital. Mussa (1977) and Abel (1983) provided formal 
underpinnings of the $q$-theory of investment in optimizing models that feature convex costs of adjusting the capital stock. This formal analysis reflects the distinction between average $q$, which is the ratio defined by Brainard and Tobin, and marginal $q$, which is the ratio of the marginal valuation of an additional unit of capital to the cost of that additional unit of capital. In the convex adjustment cost framework, the optimal rate of investment equates the marginal cost of investment, consisting of the purchase price plus the marginal adjustment cost, with marginal $q$. However, marginal $q$ is not directly observable. Hayashi (1982) provided conditions under which marginal $q$ and average $q$ are equal, thereby allowing researchers to use average $q$, which is observable, as a proxy for marginal $q$, which is the appropriate variable to be equated with the marginal cost of investment.

The Hayashi conditions are (1) current operating profits, after maximizing over any variable factors of production, such as labor, are proportional to the capital stock; and (2) the adjustment cost function is linearly homogeneous in investment and the capital stock. The first of these two conditions would be satisfied by a perfectly competitive firm with a production function that is linearly homogeneous in capital and the variable factors of production. Implicit in most invocations of the Hayashi conditions is the assumption that the firm does not face any financial constraints.

A powerful implication of the $q$-theory of investment derived in an adjustment cost framework without financial constraints is that marginal $q$ is a sufficient statistic for investment. Under the Hayashi conditions, marginal $q$ and average $q$ are identically equal, so average $q$, which is the observable construct used in much empirical work, is thus a sufficient statistic for investment. FHP (1988) showed that, empirically, average $q$ is not a sufficient statistic for investment for firms that are likely to be financially constrained, such as firms that do not pay dividends and firms that are growing rapidly. FHP found that for such firms, cash flow has a positive effect on investment, even taking account of the supposedly-sufficient statistic, average $q$. They interpret their finding as evidence that financial constraints impact capital investment by these firms.

FHP's interpretation of the positive coefficient on cash flow has faced three types of challenges. Kaplan and Zingales (1997) challenged FHP's reliance on dividend-payout ratios to categorize firms into three classes. FHP Class I firms have the lowest dividend-payout ratios and are regarded by FHP as likely to be financially constrained. Kaplan and Zingales developed an alternative classification scheme based on various criteria gleaned from financial statements and other documents. Contrary to FHP, they find evidence that cash-flow sensitivities are highest for firms that are the least financially constrained. 
A second type of challenge, that includes Gomes (2001), Cooper and Ejarque (2003), Alti (2003) and Abel and Eberly (2011), has produced theoretical models for which an investment regression on average $q$ and cash flow would yield a positive coefficient on cash flow, even though the models do not incorporate financial constraints. In these models, none of which satisfy the Hayashi conditions, a positive coefficient on cash flow cannot be viewed as evidence of financial constraints.

A third type of challenge is based on measurement error in $q$. In a published comment that appears in the same volume as FHP, Poterba (1988) states "If for any of these reasons the measured $Q$ variable provides an error-ridden indicator of the firm's true prospects, then econometric results may find that current cash flow affects investment only because this variable, just like measured $Q$, is correlated with the 'true' marginal $q$ variable that firms consider in making investment decisions." (p. 202) Whited (1992) developed an alternative test for the impact of financial constraints based on estimating an Euler equation associated with a firm's investment decision. The Euler equation fits the data well for firms that are not financially constrained but not for firms that are financially constrained, consistent with investment being affected by financial constraints. She reiterated the point that a positive coefficient on cash flow might arise from measurement error in $q,{ }^{4}$ and this point was further echoed by Gilchrist and Himmelberg $(1995)^{5}$ and in a survey article by Roberts and Whited $(2013)^{6}$. Erickson and Whited (2000) addressed the measurement error head on by developing and applying consistent higher-order moment estimators. More recently, Abel (2018) developed a dynamic model of the firm that satsifies the Hayashi conditions and does not face financial constraints. For such a firm, average $q$ is identically equal to marginal $q$. Abel derived closed-form expressions for the plims of the estimated coefficients on $q$ and cash flow in investment regressions, and showed analytically that classical measurement error in $q$ will lead to a positive coefficient on cash flow, even though there are no financial constraints.

The equality of average $q$ and marginal $q$ is a crucial assumption for empirical analyses that relate the investment-capital ratio to average $q$. However, even for perfectly competitive

\footnotetext{
${ }^{4}$ Whited (1992), p. 1426, observed "cash flow may not be picking up the desired liquidity effect but may be proxying either for an accelerator effect or for information about future investment opportunities not captured by $q . "$

${ }^{5}$ Gilchrist and Himmelberg (1995), p. 544, state "More generally, anything that systematically reduces the signal-to-noise ratio of Tobin's Q (for example, measurement error or 'excess volatility' of stock prices) will shift explanatory power away from Tobin's Q toward cash flow, thus making such firms appear to be financially constrained when in fact they are not.

${ }^{6}$ Roberts and Whited (2013), p. 498, state that because of the high positive correlation between Tobin's $q$ and cash flow, "the coefficient on cash flow, $\beta_{j}$, is biased upwards. Therefore, even if the true coefficient on cash flow is zero, the biased OLS coefficient can be positive."
} 
firms with constant returns to scale in their production and adjustment cost functions, measured average $q$ can differ from marginal $q$, which is equated to the marginal cost of investment. Ideally, average $q$ would measure the contribution to firm value of its physical capital stock. However, the value of the firm, which is the numerator of average $q$, can include the values of other assets, such as inventories, remaining depreciation tax shields on existing capital, intangible assets, and financial assets, such as securities and cash on hand. The values of these other assets are often subtracted from the total value of the firm in constructing the numerator of average $q$. FHP, Whited (1992), and Peters and Taylor (2017) subtract inventories from firm value in computing average $q$. In the seminal demonstration of the equality of marginal $q$ and average $q$, Hayashi (1982) subtracts the present value of future tax savings due to depreciation deductions accruing to existing capital, and a similar correction is employed by Summers (1981) and Hoshi and Kashyap (1990).

Hayashi and Inoue (1991) consider a firm with multiple capital goods. Under linear homogeneity of the production and adjustment cost functions, the value of a perfectly competitive firm, $V\left(K_{1}, \ldots, K_{n}\right)$, would be linearly homogeneous in the $n$ types of capital, so that $V=\sum_{i=1}^{n} V_{K_{i}} K_{i}$, where $V_{K_{i}}$ is the marginal valuation of type $i$ capital. The replacement cost of the total capital stock is $R C \equiv \sum_{i=1}^{n} p_{i} K_{i}$, where $p_{i}$ is the replacement cost of a unit of type $i$ capital. Marginal $q$ for type $i$ capital is $\frac{V_{K_{i}}}{p_{i}}$. If it turns out that $\frac{V_{K_{i}}}{p_{i}}$ is equal for all types $i$, then average $q, \frac{V}{R C}$, would equal this common value of marginal $q$. Peters and Taylor (2017) treat intangible capital (arising from R\&D investment and a portion of selling, general and administrative expense) as a second type of capital. They impose conditions that make marginal $q$ for physical capital and marginal $q$ for intangible capital identically equal to each other. Among these conditions is the assumption that physical capital and intangible capital are perfect substitutes in production. While this assumption may be plausible for physical capital and intangible capital, it would not be appropriate in the context of our analysis, where the two types of capital are physical capital and cash on hand.

It might seem that it is straightforward to adjust the value of the firm for its holding of cash on hand, since cash is easier to value than, say, inventories, or the present value of future depreciation tax shields in an uncertain tax environment. Thus, for instance, Hoshi and Kashyap (1990) and Peters and Taylor (2017) subtract cash from the market value of the firm in the numerator of Tobin's $q$. Reducing the firm's value dollar-for-dollar for each dollar of cash on hand is the correct adjustment in the absence of financial constraints. However, in the presence of financial constraints, the value of a dollar of cash on hand can 
exceed one dollar. In this case, with the marginal value of a dollar of cash greater than one, the correct adjustment to the firm's value would be to subtract the amount of cash on hand, multiplied by the marginal value of cash. However, the marginal value of cash is not directly observable, except when the firm is paying dividends.

Bolton et al. (2011) emphasized the importance of the marginal value of cash in the investment decision. They developed a continuous-time model to analyze the investment and liquidity management decisions of a firm that has costly access to external finance. Since the use of external finance is costly, the shadow value of a dollar of cash of hand can exceed a dollar. Put differently, the use of a dollar of cash to purchase and install capital can have a cost that exceeds one. They showed that the standard $q$-theoretic condition that equates the marginal cost of investment and the marginal valuation of a unit of installed capital needs to be adjusted for the additional cost reflected in the shadow price of cash. Our analysis also highlights that financial constraints increase the marginal value of cash on hand and the consequent implication for optimal investment. Bolton, Chen, and Wang included a rich set of opportunities for external finance and characterized features of optimal investment and financial policy, though they did not derive closed-form solutions, nor did they address the wedge between average $q$ and marginal $q$ caused by financial constraints. In contrast, we do not allow external finance, which allows us to derive analytic solutions for optimal investment and dividend policy, even though, unlike in Bolton, Chen, and Wang, profitability is serially correlated. These solutions allow us to analyze the wedge between average $q$ and marginal $q$ and the consequent effects on investment regressions. In addition, an important difference between their model and ours is that in their model marginal $q$ is constant in the absence of financial constraints because they assume that profitability is i.i.d. By assuming persistent profitability, we can study investment regressions in a framework where marginal $q$ varies both because of exogenous profitability shocks and the time-varying tightness of financial constraints. The dependence of marginal $q$ on profitability shocks, as well as on the tightness of financial constraints, becomes important in our analysis of investment regressions.

Abel and Panageas (2019) analyze the cash management problem of a firm that faces an exogenous stochastic stream of cash flow from operations. In that framework, there is no investment decision and the capital stock is assumed to be constant over time. Since there is no investment, the cash management decision is simply a payout decision amounting to the determination of how much cash to retain within the firm and how much cash to pay out as dividends. Abel and Panageas assume that the salvage value of the firm is zero and derive a closed-form solution to characterize optimal payout policy. The current paper goes beyond 
the analysis in Abel and Panageas in two ways. First, we introduce a capital investment decision, so cash will have three potential uses: investment expenditures, dividends, and accumulation of cash on hand, which allows us to analyze various investment regressions. Second, we allow for a positive salvage value of the firm, which makes a closed-form solution for the optimal target level of cash unavailable. Nevertheless, we derive analytic results about the impact of salvage value on firm valuation and optimal investment.

\section{Profitability and Capital}

Consider a firm in continuous time. It is owned by risk-neutral shareholders with rate of time preference $\rho>0$. Profit from operations at time $t$ is $\phi_{t} K_{t}$, where $K_{t}>0$ is the capital stock at time $t$ and $\phi_{t}$ is profitability per unit of capital at time $t$. Profitability, $\phi_{t}$, is governed by a Markov regime-switching process with two regimes, $H$ and $L$. In Regime $H$, $\phi_{t}=\phi^{H}>\rho>0$. In Regime $L, \phi_{t}=\phi^{L}<0$. The negative profitability in Regime $L$ could arise from some unavoidable cost of maintaining each unit of capital. ${ }^{7}$ When Regime $H$ prevails, there is an instantaneous hazard rate $\mu^{L}>0$ that the regime switches to Regime $L$. When Regime $L$ prevails, there is an instantaneous hazard rate $\mu^{H}>0$ that the regime switches to Regime $H$.

The firm can change its capital stock over time by undertaking gross investment at rate $I_{t}$, which can be positive, negative, or zero. For simplicity, we assume that capital does not depreciate, so net investment is

$$
\dot{K}_{t}=i_{t} K_{t}
$$

where $i_{t} \equiv \frac{I_{t}}{K_{t}} \geq 0$. The total expenditure on gross investment has two components. The first component is $I_{t}$, which is the cost of purchasing uninstalled capital at a price normalized to one. If gross investment is negative, then $-I_{t}=-i_{t} K_{t}>0$ represents the gross proceeds from selling $I_{t}$ units of capital. The second component is the adjustment cost, $c\left(i_{t}\right) K_{t}$, which is a linearly homogeneous function of $I_{t}$ and $K_{t}$. To be consistent with much of the

\footnotetext{
${ }^{7}$ For instance, instantaneous profit could be $\max _{L} p F(K, L)-w L-m K$, where $p$ is the exogenous price of the firm's output, $L$ is labor, $w$ is the exogenous wage rate of labor, $m$ is the exogenous maintenance cost per unit of capital, and the function $F(K, L)$ is linearly homogenous in $K$ and $L$, with $F(K, 0)=0$. Profit can be rewritten as $\left(\max _{l}[p f(l)-w l]-m\right) K$, where $l \equiv L / K$ and $f(0)=0$. Equivalently, profit is $\phi K$, where $\phi \equiv \max _{l}[p f(l)-w l]-m$, which will be negative for sufficiently small $p>0$, even though $\max _{l}[p f(l)-w l] \geq 0$ for any $p>0$.
} 
literature on investment and $q$, we assume that the adjustment cost function is quadratic in i. Specifically,

$$
c(i)=\frac{1}{2} \theta i^{2},
$$

where $\theta>0$ is the adjustment cost scale factor. Note that the adjustment cost is positive for any nonzero rate of investment. Therefore, if gross investment is negative, that is, if the firm sells $-i_{t} K_{t}>0$ units of capital, its net proceeds from the sale of capital are reduced by the amount of the adjustment cost $\frac{1}{2} \theta i_{t}^{2} K_{t}$.

We impose the restriction

$$
\theta>\frac{1}{-2 \phi^{L}}>0
$$

which implies that the net proceeds from selling capital, $-\left(i+\frac{1}{2} \theta i^{2}\right) K$, must be less than $\left|\phi^{L}\right|$. That is, the firm cannot meet its required payments of $-\phi^{L} K>0$ by selling capital. ${ }^{8}$ This restriction on $\theta$ does not imply that negative gross investment is never optimal. Indeed, as we will show, in the presence of financial constraints, optimal gross investment will be negative in Regime $L$ when the firm's cash on hand is very low.

We allow for the possibility that the firm terminates at some date, either because future prospects are so unfavorable that the firm chooses to terminate or because financial constraints force the firm to terminate in a way we discuss in Section 3. Upon termination for whatever reason, the shareholders of the firm receive a salvage value $\alpha K$, where $0 \leq \alpha<1$.

The firm operates in continuous time but it is convenient to treat each episode in which Regime $H$ or Regime $L$ continuously prevails as a single period in a quasi-discrete-time framework. The lengths of the time periods are random and are generated by the Markov regime-switching process for profitability. We define three terms below to represent the expected present value of cash flow, $\phi_{t}$, over a single regime and over the entire sequence of future regimes.

\section{Definition 1 Define}

1. $\Phi^{H} \equiv \frac{\phi^{H}}{\rho+\mu^{L}}>0 \quad$ (Myopic value of Regime $H$ )

\footnotetext{
${ }^{8}$ The net flow of proceeds from selling capital is $\psi(i) K$, where $\psi(i) \equiv-\left(i+\frac{1}{2} \theta i^{2}\right)$. Use $\psi^{\prime}(i)=$ $-(1+\theta i)$, and $\psi^{\prime \prime}(i)=-\theta<0$ to obtain $\arg \max _{i} \psi(i)=-\frac{1}{\theta}$ and $\max _{i} \psi(i)=-\left(-\frac{1}{\theta}+\frac{1}{2} \theta\left(-\frac{1}{\theta}\right)^{2}\right)=\frac{1}{2 \theta}$. Therefore, if $\theta>\frac{1}{-2 \phi^{L}}>0$, then the proceeds from selling capital, $\psi(i) K$, are smaller than $-\phi^{L} K>0$, the required payment in Regime $L$.
} 
2. $\Phi^{L} \equiv \frac{\phi^{L}}{\rho+\mu^{H}}<0 \quad$ (Myopic value of Regime $\left.L\right)$

3. $\Gamma \equiv \frac{\mu^{L}}{\rho+\mu^{L}} \frac{\mu^{H}}{\rho+\mu^{H}}<1 \quad$ (Roundtrip discount factor)

The myopic value of Regime $H, \Phi^{H} \equiv \frac{\phi^{H}}{\rho+\mu^{L}}$, is the expected present value of profitability $\phi_{t}$ over the duration of a single Regime $H$. We refer to this valuation as myopic because it ignores any profitability after the particular Regime $H$ ends. Similarly, the myopic value of Regime $L, \Phi^{L} \equiv \frac{\phi^{L}}{\rho+\mu^{H}}$, is the expected present value of profitability $\phi_{t}$ over the duration of a single Regime $L$. The roundtrip discount factor $\Gamma \equiv \frac{\mu^{L}}{\rho+\mu^{L}} \frac{\mu^{H}}{\rho+\mu^{H}}$ is the expected present value of a dollar to be received upon the first return to the currently-prevailing regime after the first occurrence of the other regime. ${ }^{9}$

Definition 1 is useful in obtaining simple expressions for conditional expected present values of profitability over the infinite future. Specifically,

$$
\chi^{H} \equiv \frac{1}{1-\Gamma}\left[\Phi^{H}+\frac{\mu^{L}}{\rho+\mu^{L}} \Phi^{L}\right]=E\left\{\int_{t}^{\infty} \phi_{s} e^{-\rho(s-t)} d s \mid \phi_{t}=\phi^{H}\right\}
$$

and

$$
\chi^{L} \equiv \frac{1}{1-\Gamma}\left[\Phi^{L}+\frac{\mu^{H}}{\rho+\mu^{H}} \Phi^{H}\right]=E\left\{\int_{t}^{\infty} \phi_{s} e^{-\rho(s-t)} d s \mid \phi_{t}=\phi^{L}\right\} .
$$

Observe that $\chi^{H}-\chi^{L}=\frac{1}{1-\Gamma}\left(\frac{\rho}{\rho+\mu^{H}} \Phi^{H}-\frac{\rho}{\rho+\mu^{L}} \Phi^{L}\right)>0$. The present values $\chi^{H}$ and $\chi^{L}$ are useful in Section 3.1 in characterizing the value of the firm in the absence of financial constraints.

\section{A Financially Constrained Firm}

In this section we introduce financial constraints and analyze their impact on optimal investment and payout policy. To make the financial constraints as stark as possible, we assume that an ongoing firm cannot raise any external funds. That is, the firm cannot borrow, issue new equity, or pay negative dividends. When the firm is in Regime $L$, it must make required payments, $-\phi^{L} K>0$, and can use previously accumulated cash on hand and any proceeds, net of adjustment costs, from uninstalling and selling some of its capital. The

\footnotetext{
${ }^{9}$ Suppose that Regime $H$, say, prevails at time $t$ and continues to prevail until time $t_{1}>t$ when Regime $L$ arrives. Regime $L$ prevails until time $t_{2}>t_{1}$, when a new Regime $H$ arrives. The roundtrip discount factor is the expected present value as of time $t$ of a dollar to be received at time $t_{2}$. Formally, $\Gamma=E\left\{e^{-\rho\left(t_{2}-t\right)}\right\}$ $=E\left\{e^{-\rho\left(t_{2}-t_{1}\right)}\right\} E\left\{e^{-\rho\left(t_{1}-t\right)}\right\}=\frac{\mu^{H}}{\rho+\mu^{H}} \frac{\mu^{L}}{\rho+\mu^{L}}$.
} 
restriction on the adjustment cost scale parameter $\theta$ in equation (3) implies that the flow of net proceeds from selling capital cannot be large enough to pay the entire $-\phi^{L} K>0$. Therefore, the firm must use some cash on hand to make this required payment. If the firm has zero cash on hand, it will not be able to make the required payment and will have to terminate permanently. ${ }^{10}$ In the event of termination, shareholders lose their claim on any future positive cash flows but receive the salvage value $\alpha K \geq 0$. The prospect of forced termination provides an incentive for the firm to accumulate cash. However, the preference of shareholders to receive dividends earlier rather than later limits the amount of cash that the firm wants to carry on hand.

Let $X_{t}$ be the amount of cash on hand at time $t$. The equality of sources and uses of cash can be written as

$$
\phi_{t} K_{t}=\dot{X}_{t}+D_{t}+\left(i_{t}+c\left(i_{t}\right)\right) K_{t}
$$

The source of cash on the left hand side of equation (6) is net profits from operations, $\phi_{t} K_{t}$. The uses of cash on the right hand side of equation are (1) accumulation of cash on hand, $\dot{X}_{t}$; (2) payment of dividends, $D_{t}$; and (3) expenditure on investment, $\left(i_{t}+c\left(i_{t}\right)\right) K_{t}$, which includes both the purchase price of capital and the adjustment cost. A negative value of $X$ would be equivalent to interest-free debt owed by the firm, and a negative value of $D$ would be equivalent to issuing equity. Since we do not allow the firm to issue debt or equity, the financial constraints can be written as $X_{t} \geq 0$ and $D_{t} \geq 0$.

With the financial constraints facing the firm, the stock of cash on hand, $X_{t}$, becomes an additional state variable. The value function of the firm in Regime $H, V^{H}(K, X)$, satisfies

$$
\rho V^{H}(K, X)=\max _{D, i} D+V_{X}^{H}(K, X) \dot{X}+V_{K}^{H}(K, X) \dot{K}+\mu^{L}\left(V^{L}(K, X)-V^{H}(K, X)\right)
$$

The maximand on the right hand side of equation (7) is the dividend, $D$, plus the capital gain, $\frac{1}{d t} E\{d V\}$, which reflects the change in cash, $\dot{X}$, the change in the capital stock, $\dot{K}$, and the expected change in value associated with a change in regime.

Lemma $1 V_{X}^{j}(X, K) \geq 1$, for $j \in\{H, L\}$.

Lemma 1 simply states that increasing a firm's cash on hand, $X$, by one dollar will increase the firm's value by at least one dollar because that additional dollar can be paid

\footnotetext{
${ }^{10}$ As shown in footnote 8 , the maximal flow of net proceeds from negative gross investment is $\frac{1}{2 \theta} K$. In this section we introduce the assumption that $\theta$ is arbitrarily large, so the maximal flow of net proceeds from selling capital is arbitrarily small.
} 
immediately to shareholders and leave the value of the firm unchanged from its initial value before receiving the dollar.

Lemma 1 and the constraint $D \geq 0$ imply $\max _{D}\left(1-V_{X}(K, X)\right) D=0$ so the ODE in equation (7), using equations (1) and (6), becomes

$$
\begin{aligned}
\rho V^{H}(K, X)= & \max _{i} V_{X}^{H}(K, X)\left(\phi^{H}-(i+c(i))\right) K+V_{K}^{H}(K, X) i K \\
& +\mu^{L}\left(V^{L}(K, X)-V^{H}(K, X)\right) .
\end{aligned}
$$

The value function $V^{H}(K, X)$ is linearly homogeneous in $K$ and $X$, so it is convenient to write the value function as

$$
V^{H}(K, X)=v^{H}(x) K,
$$

where $x_{t} \equiv \frac{X_{t}}{K_{t}} \geq 0$ is the cash-to-capital ratio. The marginal values of cash and capital are, respectively,

$$
V_{X}^{H}(K, X)=v_{x}^{H}(x)
$$

and

$$
V_{K}^{H}(K, X)=v^{H}(x)-x v_{x}^{H}(x) .
$$

Divide the ODE in equation (8) by $K$ and use equations (9)-(11) to obtain

$$
\begin{aligned}
\rho v^{H}(x)= & \max _{i} v_{x}^{H}(x)\left(\phi^{H}-(i+c(i))\right)+\left(v^{H}(x)-x v_{x}^{H}(x)\right) i \\
& +\mu^{L}\left(v^{L}(x)-v^{H}(x)\right) .
\end{aligned}
$$

To solve the maximization on the right hand side of equation (12), differentiate the right hand side of this equation with respect to $i$, and set this derivative equal to zero to obtain the following first-order condition for investment

$$
1+c^{\prime}(i)=\frac{v^{H}(x)}{v_{x}^{H}(x)}-x .
$$

The right hand side of equation (13) is the ratio of the marginal value of capital, $V_{K}^{H}$, to the marginal value of cash on hand, $V_{X}^{H}$, which is marginal $q,{ }^{11}$

$$
q_{M}^{H}(x) \equiv \frac{v^{H}(x)}{v_{x}^{H}(x)}-x .
$$

\footnotetext{
${ }^{11}$ The first-order condition in equation (13) can be written as $1+c^{\prime}\left(i^{H}\right)=\frac{V_{K}^{H}(K, X)}{V_{X}^{H}(K, X)}$. Bolton et al. (2011), p. 1546, express this first-order condition as "marginal cost of investment $=($ marginal $q) /($ marginal cost of financing)," where their marginal $q$ is $V_{K}(K, X)$. Our definition of marginal $q$ in equation (14) incorporates
} 
Use the definition of $q_{M}^{H}(x)$ from equation (14) in the first-order condition in equation (13) and the specification of the quadratic adjustment cost function in equation (2) to obtain the optimal investment-capital ratio in Regime $H$

$$
i^{H}(x)=\frac{q_{M}^{H}(x)-1}{\theta} .
$$

Equation (15) is the standard $q$ theory of investment with quadratic adjustment costs. The optimal investment-capital ratio, $i$, is a linear function of marginal $q$ with slope $\frac{1}{\theta}$. As shown in equation (25) below, marginal $q$ is an increasing function of $x$. Therefore, equation (15) implies that the optimal investment-capital ratio is an increasing function of cash on hand, $x$. It is noteworthy that the impact of $x$ on the optimal investment-capital ratio $i^{H}(x)$ operates entirely through $q_{M}^{H}(x)$. Given the value of $q_{M}^{H}$, the amount of cash on hand, $x$, has no independent effect on optimal investment, that is, marginal $q$ is a sufficient statistic for optimal investment. However, marginal $q, q_{M}^{H}(x)$, is not directly observable because $V_{K}(K, X), V_{X}(K, X)$, and $\frac{V_{K}(K, X)}{V_{X}(K, X)}$ are all unobservable. In Section 4, we analyze the relationship between average $q$, which is observable, and marginal $q$, which is not observable.

In order to obtain closed-form solutions for the value of the firm in both regimes, we focus on a limiting case of the adjustment cost function $c(i) K=\frac{1}{2} \theta i^{2} K$ in which the scale parameter $\theta$ approaches infinity. Thus, for a given value of $q_{M}^{H}(x)$, equation (15) implies that $\lim _{\theta \rightarrow \infty} i^{H}(x)=0$ and $\lim _{\theta \rightarrow \infty} c\left(i^{H}(x)\right)=\lim _{\theta \rightarrow \infty} \frac{1}{2} \theta\left(\frac{q_{M}^{H}(x)-1}{\theta}\right)^{2}=0$. Remarkably, as the adjustment cost scale factor $\theta$ becomes arbitarily large, the adjustment cost evaluated at the optimal investment-capital ratio, $c\left(i^{H}(x)\right)$, approaches zero and hence the total cost of investment $\left[i^{H}(x)+c\left(i^{H}(x)\right)\right] K$ also approaches zero. Nevertheless, the marginal adjustment cost, $c^{\prime}\left(i^{H}(x)\right)=\theta i$, approaches the given value $q_{M}^{H}(x)-1$, which is finite and, except when $q_{M}^{H}(x)=1$, nonzero. That is, for a given value $q_{M}^{H}(x)-1, i$ approaches zero at the same rate that $\theta$ approaches infinity, so that the product $\theta i$ approaches a constant. The optimal value of $\theta i$ is an increasing linear function of $q_{M}^{H}(x)$, and hence for a sufficiently large, but fixed, value of $\theta$, the optimal investment-capital ratio $i$ is an increasing linear function of $q_{M}^{H}(x)$. The main benefit of focusing on the limiting case where $\theta$ becomes large is that we can ignore the impact of adjustment costs on marginal $q$. This simplification allows us to solve for marginal $q$ explicitly, as we show next.

$V_{X}(K, X)$ into the denominator, so that the first-order condition in equation (13) is $1+c^{\prime}\left(i^{H}\right)=q_{M}^{H}(x)$, which simplifies the equation for optimal investment in equation (15). It is a definitional matter whether to define marginal $q$ as $V_{K}(K, X)$ or $\frac{V_{K}(K, X)}{V_{X}(K, X)}$, neither of which is directly observable. With either definition, average $q$ is generally not equal to marginal $q$ in the presence of financial constraints, as discussed in Section 4 . 
For suffciently large $\theta$, the terms $i^{H}(x)$ and $c\left(i^{H}(x)\right)$ are vanishingly small so that the ODE in equation (12) approaches $\rho v^{H}(x)=v_{x}^{H}(x) \phi^{H}+\mu^{L}\left(v^{L}(x)-v^{H}(x)\right)$, which, after dividing both sides by $\rho+\mu^{L}$, becomes

$$
v^{H}(x)=v_{x}^{H}(x) \Phi^{H}+\frac{\mu^{L}}{\rho+\mu^{L}} v^{L}(x) .
$$

Similarly, in Regime $L$

$$
v^{L}(x)=v_{x}^{L}(x) \Phi^{L}+\frac{\mu^{H}}{\rho+\mu^{H}} v^{H}(x) .
$$

The ODEs in equations (16) and (17) hold for non-negative values of the cash-to-capital ratio $x$ that are small enough that it is optimal not to pay dividends. If Regime $H$ prevails long enough, then eventually cash on hand reaches a level $x^{*}$ at which the marginal value of a dollar of cash, $v_{x}(x)$, equals one. When cash on hand reaches $x^{*}$, the firm maintains $x=x^{*}$ in Regime $H$ by paying out positive cash flow (net of investment expenditures, which are arbitrarily small) as dividends.

Lemma $2 x^{*}>0$ if and only if $v^{L}\left(x^{*}\right)>\alpha$.

The only reason for the firm to hold cash is to be able to survive, for at least a while, when the next Regime $L$ arrives. Lemma 2 states that this opportunity to survive when Regime $L$ arrives is worthwhile only if the value of the firm when it holds $x^{*} K$ exceeds the salvage value $a K$ when Regime $L$ arrives.

The critical value $x^{*}$ is characterized by the following two boundary conditions

$$
v_{x}^{H}\left(x^{*}\right)=1
$$

and

$$
v_{x x}^{H}\left(x^{*}\right)=0
$$

In Regime $L$, the firm uses cash on hand to make required cash outflows. However, if Regime $L$ prevails long enough, cash on hand eventually falls to zero. When that happens, the firm is forced to terminate immediately and permanently and shareholders receive the salvage value $\alpha K$, as reflected in the following boundary condition

$$
v^{L}(0)=\alpha
$$


The boundary conditions in equations (18) and (19) imply that a firm will never choose to accumulate cash to a level higher than $X=x^{*} K{ }^{12}$ Therefore, we confine attention to $x \in\left[0, x^{*}\right]$. We present the distribution of the endogenous variable $x$ in Section 4.1.

Appendix B solves the ODEs in equations (16) and (17) along with the boundary conditions conditions in equations (18), (19), and (20) in the case in which $v^{L}\left(x^{*}\right) \geq \alpha$ so that the firm chooses to continue operating when Regime $L$ arrives, at least for some values of $x$. This appendix presents expressions for $v^{H}(x)$ and $v^{L}(x)$ that are closed-form functions of the fundamental parameters $\left(\rho, \phi^{H}, \phi^{L}, \mu^{H}, \mu^{L}\right.$ and the resulting $\Phi^{H}, \Phi^{L}$, and $\left.\Gamma\right)$ and $x^{*}$. It also presents $x^{*}$ as the root of a nonlinear equation; in the special case with zero salvage value, i.e., $\alpha=0$, this appendix presents a closed-form solution for $x^{*}$. Appendix B also shows that $v^{H}(x)$ and $v^{L}(x)$ are both concave on $\left[0, x^{*}\right]$.

\subsection{The Valuation of the Firm in the Absence of Financial Con- straints}

Consider a firm that does not face any financial constraint. Let $\widetilde{v}^{j}$ be the value of this firm per unit of capital in Regime $j$ when it has zero cash on hand. More generally, when cash on hand can be positive, the value of the firm per unit of capital is $\widetilde{v}^{j}+x$. Therefore, the analogue of $v_{x}^{j}(x)$ in equations (16) and (17) is identically equal to one so evaluating these equations at $x=0$ yields

$$
\widetilde{v}^{H}=\Phi^{H}+\frac{\mu^{L}}{\rho+\mu^{L}} \widetilde{v}^{L}
$$

and

$$
\widetilde{v}^{L}=\Phi^{L}+\frac{\mu^{H}}{\rho+\mu^{H}} \widetilde{v}^{H} .
$$

This two-equation linear system has the solution $\widetilde{v}^{j}=\chi^{j}$ for $j \in\{H, L\}$, where $\chi^{j}$ is the expected present value of the infinite stream of profitability conditional on currently being in Regime $j$ (see equations (4) and (5)). However, the value of the firm must obey $\widetilde{v}^{L} \geq \alpha$, which is analogous to the boundary condition in equation (22). The following proposition summarizes the valuation of firm in the absence of financial constraints.

\footnotetext{
${ }^{12}$ If the firm started its existence in Regime $H$ with $X>x^{*} K$, it would immediately pay a dividend of $X-x^{*} K$. Once the value of $x$ is less than or equal to $x^{*}$, it will remain less than or equal to $x^{*}$ for the remainder of the firm's life.
} 
Proposition 1 In the absence of financial constraints, the value of the firm per unit of capital in each regime is

$$
\begin{aligned}
& \widetilde{v}^{H}=\max \left\{\chi^{H}, \Phi^{H}+\frac{\mu^{L}}{\rho+\mu^{L}} \alpha\right\}=\max \left\{\frac{1}{1-\Gamma}\left[\Phi^{H}+\frac{\mu^{L}}{\rho+\mu^{L}} \Phi^{L}\right], \Phi^{H}+\frac{\mu^{L}}{\rho+\mu^{L}} \alpha\right\} \\
& \widetilde{v}^{L}=\max \left\{\chi^{L}, \alpha\right\}=\max \left\{\frac{1}{1-\Gamma}\left[\Phi^{L}+\frac{\mu^{H}}{\rho+\mu^{H}} \Phi^{H}\right], \alpha\right\} .
\end{aligned}
$$

Consider two cases. Case I: $\chi^{L}<\alpha$. If $\chi^{L}<\alpha$, then the firm would choose to terminate as soon as Regime $L$ arrives and the shareholders would receive $\alpha K$ upon termination of the firm. In this case, the firm would remain alive for only a single Regime $H$ so $\widetilde{v}^{L}=\alpha$ and $\widetilde{v}^{H}=\Phi^{H}+\frac{\mu^{L}}{\rho+\mu^{L}} \alpha{ }^{13} \quad$ Case II: $\quad \chi^{L} \geq \alpha$. In this case, the firm would not choose to terminate in Regime $L$. In Regime $L$, the firm's value per unit of capital would be $\chi^{L}$ and the firm would operate forever (it would not terminate in Regime $H$, since $\chi^{H}>\chi^{L}$ ). Since the firm would operate forever, $\widetilde{v}^{L}=\chi^{L}$ and $\widetilde{v}^{H}=\chi^{H}$.

\subsection{Optimal Investment and Marginal $q$}

In this model with quadratic adjustment costs, the optimal investment-capital ratio is a linear function of marginal $q$, with slope $\frac{1}{\theta}>0$, as shown for Regime $H$ in equation (15). A similar expression holds in Regime $L$, so that the optimal investment-capital ratio in each regime is

$$
i^{j}(x)=\frac{q_{M}^{j}(x)-1}{\theta}, \text { for } j \in\{H, L\}
$$

where

$$
q_{M}^{j}(x) \equiv \frac{v^{j}(x)}{v_{x}^{j}(x)}-x, \text { for } j \in\{H, L\} .
$$

Differentiating $q_{M}^{j}(x)$ in equation (24) with respect to $x$ yields

$$
\frac{d q_{M}^{j}(x)}{d x}=-\frac{v^{j}(x)}{\left[v_{x}^{j}(x)\right]^{2}} v_{x x}^{j}(x)>0, \quad \text { for } 0 \leq x<x^{*} \text { and } j \in\{H, L\} .
$$

Therefore, in both regimes, marginal $q$ is strictly increasing in $x$ on the domain $\left[0, x^{*}\right)$.

\footnotetext{
${ }^{13}$ The firm would not choose to terminate in Regime $H$ because $\widetilde{v}^{H}-\alpha=\Phi^{H}+\frac{\mu^{L}}{\rho+\mu^{L}} \alpha-\alpha=$ $\frac{1}{\rho+\mu^{L}}\left(\phi^{H}-\rho \alpha\right)>0$, where the inequality follows from the assumptions that $\phi^{H}>\rho>0$ and $\alpha<1$.
} 
Now evaluate $v^{j}(x)$ and $q_{M}^{j}(x)$ at $x=0$ and $x=x^{*}$, where these functions attain their minimum and maximum values, respectively, in each regime. Propositions 2 and 3, which present these values at $x=0$ and $x=x^{*}$, respectively, will be useful in Section 4 when we compare average $q$ and marginal $q$.

First consider $v^{j}(0)$ and $q_{M}^{j}(0)$ for $j \in\{H, L\}$.

Proposition 2 Suppose that $x^{*}>0$. Then

$$
\begin{aligned}
& \text { 1. } v^{H}(0)=v_{x}^{H}(0) \Phi^{H}+\frac{\mu^{L}}{\rho+\mu^{L}} \alpha>\Phi^{H} \\
& \text { 2. } q_{M}^{H}(0)=\Phi^{H}+\frac{\mu^{L}}{\rho+\mu^{L}} \frac{\alpha}{v_{x}^{H}(0)} \geq \Phi^{H} \\
& \text { 3. } v^{L}(0)=\alpha \\
& \text { 4. } q_{M}^{L}(0)=\frac{\alpha}{v_{x}^{L}(0)} \leq \alpha
\end{aligned}
$$

Equation (23) implies that optimal investment when $x=0$ will be positive if and only if marginal $q, q_{M}^{j}(0)>1$. Statement 2 of Proposition 2 implies that if $\Phi^{H}>1$, then the optimal investment-capital ratio, $i^{H}(0)$, is positive. If $\Phi^{H}>1$, then even if the firm were to terminate at the end of the current Regime $H$, the expected present value of operating profits accruing to a unit of new capital, $\Phi^{H}$, would be larger than the cost of adding that unit of capital. Thus, optimal investment in Regime $H$ is positive if $\Phi^{H}>1$.

Since the salvage value per unit of capital, $\alpha$, is less than one, Statement 4 of Proposition 2 implies that $q_{M}^{L}(0)<1$, so equation (23) implies that in Regime $L$ optimal investment is negative when $x=0$. Negative gross investment involves selling capital. However, the restriction in equation (3) implies that the proceeds, net of adjustment costs, from selling capital will not be sufficient to pay the required outflow per unit of capital in Regime $L$, $-\phi^{L}>0$. Therefore, when Regime $L$ prevails, the ratio of cash to the capital stock, $x$, will be declining ${ }^{14}$ and as soon as $x$ reaches zero, the firm will be forced to terminate. The shareholders receive the salvage value $\alpha K$ when the firm terminates.

Now consider the situation in which the firm is holding its maximal ratio of cash to capital, that is, when $x=x^{*}$. As a preliminary step, it is useful to evaluate $v^{j}(x)$ and $v_{x}^{j}(x)$ in both regimes $(j=H$ and $j=L)$ when $x=x^{*}>0$. The boundary condition in equation (18) provides one of these values, namely, $v_{x}^{H}\left(x^{*}\right)=1$. To evaluate the corresponding derivative

\footnotetext{
${ }^{14}$ Even though $K$ will be declining, $x \equiv \frac{X}{K}$ will be declining because $\frac{\dot{x}}{x}=\frac{\dot{X}}{X}-\frac{\dot{K}}{K}=\frac{\dot{X}}{X}-i$ where $\frac{\dot{X}}{X}<0$ and $i$ is arbitrarily small.
} 
in Regime $L$, differentiate the ODE in equation (16) with respect to $x$ and evaluate the resulting equation at $x=x^{*}$ to obtain

$$
v_{x}^{H}\left(x^{*}\right)=v_{x x}^{H}\left(x^{*}\right) \Phi^{H}+\frac{\mu^{L}}{\rho+\mu^{L}} v_{x}^{L}\left(x^{*}\right), \quad \text { for } x^{*}>0 .
$$

Now use the boundary conditions $v_{x}^{H}\left(x^{*}\right)=1$ and $v_{x x}^{H}\left(x^{*}\right)=0$ from equations (18) and (19), respectively, in equation (26) and rearrange to obtain

$$
v_{x}^{L}\left(x^{*}\right)=\frac{\rho+\mu^{L}}{\mu^{L}}>1, \quad \text { for } x^{*}>0 .
$$

Equation (27) states that the marginal value of a dollar of cash on hand in Regime $L$ exceeds one dollar when $x=x^{*}$. Therefore, when $x=x^{*}$ in Regime $L$, it is optimal for the firm to retain all of its cash rather than pay dividends. Of course, the firm will have to use some of its cash in this situation to make the required payments $-\phi^{L} K>0$, but it is not optimal to use any of the cash to pay dividends. Since $v^{L}(x)$ is concave on the domain $\left[0, x^{*}\right], v_{x}^{L}(x)>1$ for all $x$ in $\left[0, x^{*}\right]$. Therefore, it is never optimal for the firm to pay dividends in Regime $L$.

Next calculate $v^{L}\left(x^{*}\right)$ and $v^{H}\left(x^{*}\right)$ for $x^{*}>0$. Evaluate the HJB for Regime $H$ from equation (16) at $x=x^{*}$ and use the boundary condition $v_{x}^{H}\left(x^{*}\right)=1$ from equation (18) to obtain

$$
v^{H}\left(x^{*}\right)=\Phi^{H}+\frac{\mu^{L}}{\rho+\mu^{L}} v^{L}\left(x^{*}\right) .
$$

Evaluate the HJB for Regime $L$ from equation (17) at $x=x^{*}$ and use $v_{x}^{L}\left(x^{*}\right)=\frac{\rho+\mu^{L}}{\mu^{L}}$ from equation (27) to obtain

$$
v^{L}\left(x^{*}\right)=\frac{\rho+\mu^{L}}{\mu^{L}} \Phi^{L}+\frac{\mu^{H}}{\rho+\mu^{H}} v^{H}\left(x^{*}\right) .
$$

Equations (28) and (29) are two linear equations in $v^{H}\left(x^{*}\right)$ and $v^{L}\left(x^{*}\right)$.

Proposition 3 If $x^{*}>0$, then
1. $v^{H}\left(x^{*}\right)=\frac{\Phi^{H}+\Phi^{L}}{1-\Gamma}<\widetilde{v}^{H}$
2. $q_{M}^{H}\left(x^{*}\right)=\frac{\Phi^{H}+\Phi^{L}}{1-\Gamma}-x^{*}<\widetilde{v}^{H}$
3. $v^{L}\left(x^{*}\right)=\frac{\rho+\mu^{L}}{\mu^{L}} \frac{\Phi^{L}+\Gamma \Phi^{H}}{1-\Gamma}<\widetilde{v}^{L}$
4. $q_{M}^{L}\left(x^{*}\right)=\frac{\Phi^{L}+\Gamma \Phi^{H}}{1-\Gamma}-x^{*}<\widetilde{v}^{L}$ 
Remarkably, the values of $v^{j}\left(x^{*}\right)$ and $q_{M}^{j}\left(x^{*}\right)$ are invariant to the salvage value $\alpha$. We explain this invariance in Section 5 .

Statement 3 of Proposition 3 and Lemma 2 together imply the following proposition.

Proposition $4 x^{*}>0 \Longleftrightarrow \frac{\Phi^{L}+\Gamma \Phi^{H}}{1-\Gamma}>\frac{\mu^{L}}{\rho+\mu^{L}} \alpha \Longleftrightarrow v^{H}\left(x^{*}\right)>\Phi^{H}+\frac{\mu^{L}}{\rho+\mu^{L}} \alpha$.

Proposition 4 provides two alternative, but equivalent, necessary and sufficient conditions for $x^{*}>0$. The first necessary and sufficient condition, $\frac{\Phi^{L}+\Gamma \Phi^{H}}{1-\Gamma}>\frac{\mu^{L}}{\rho+\mu^{L}} \alpha$, is expressed in terms of the primitive parameters of the firm's decision problem. The second necessary and sufficient condition, $v^{H}\left(x^{*}\right)>\Phi^{H}+\frac{\mu^{L}}{\rho+\mu^{L}} \alpha$, is a simple comparison of the value of the firm in Regime $H$ when $x=x^{*}$ and the value of the firm in Regime $H$ if it never holds any cash on hand. If the firm never holds any cash on hand, its value per unit of capital in Regime $H$ has two additive components: (1) $\Phi^{H}$, which is the expected present value of the flow of profits per unit of capital over the remainder of the current Regime $H$; and $(2) \frac{\mu^{L}}{\rho+\mu^{L}} \alpha$, which is the expected present value of the salvage value of a unit of capital that accrues to the shareholders of the firm at the end of the current Regime $H$, when the firm is forced to terminate because it cannot make the required payment $-\phi^{L} K>0$ in Regime $L$.

The firm will eventually, in finite time, experience a Regime $L$ that lasts long enough to completely exhaust its cash on hand and force it to terminate. Despite the finite lifetime of the firm, the values of $v^{H}\left(x^{*}\right)$ and $v^{L}\left(x^{*}\right)$ can be expressed as expected present values of weighted profitability over an infinite horizon, ${ }^{15}$

$$
v^{j}\left(x^{*}\right) \equiv E\left\{\int_{0}^{\infty} v_{x}^{l(t)}\left(x^{*}\right) \phi_{t} e^{-\rho t} d t \mid \phi_{0}=\phi^{j}\right\}, \quad j \in\{H, L\} .
$$

In equation (30) the value of the firm per unit of capital is the expected present value of profitability at each point in time $t$, weighted by $v_{x}^{l(t)}\left(x^{*}\right)$, the marginal value of cash at $x=x^{*}$ in the prevailing Regime $l(t)$ at time $t$. Since $v_{x}^{H}\left(x^{*}\right)=1$ and $v_{x}^{L}\left(x^{*}\right)=\frac{\rho+\mu^{L}}{\mu^{L}}>1$, the expected present values on the right hand side of equation (30) place a greater weight on $\phi^{L}<$ 0 in Regime $L$ than on $\phi^{H}>0$ in Regime $H$. The additional weight on the losses in Regime $L$ takes account of the reduction in firm value associated with the eventual termination of the firm when it runs out of cash in Regime $L$. The greater weight on negative profitabilities

\footnotetext{
${ }^{15}$ Use $v_{x}^{H}\left(x^{*}\right)=1$ and $v_{x}^{L}\left(x^{*}\right)=\frac{\rho+\mu^{L}}{\mu^{L}}>1$ to calculate $E\left\{\int_{0}^{\infty} v_{x}^{l(t)}\left(x^{*}\right) \phi_{t} e^{-\rho t} d t \mid \phi_{0}=\phi^{H}\right\}$ $=\frac{1}{1-\Gamma}\left[\Phi^{H}+\frac{\mu^{L}}{\rho+\mu^{L}} v_{x}^{L}\left(x^{*}\right) \Phi^{L}\right]=\frac{1}{1-\Gamma}\left[\Phi^{H}+\Phi^{L}\right] \quad$ and $\quad E\left\{\int_{0}^{\infty} v_{x}^{l(t)}\left(x^{*}\right) \phi_{t} e^{-\rho t} d t \mid \phi_{0}=\phi^{L}\right\}=$ $\frac{1}{1-\Gamma}\left[v_{x}^{L}\left(x^{*}\right) \Phi^{L}+\frac{\mu^{H}}{\rho+\mu^{H}} \Phi^{H}\right]=\frac{1}{1-\Gamma} \frac{\rho+\mu^{L}}{\mu^{L}}\left[\Phi^{L}+\Gamma \Phi^{H}\right]$.
} 
than on positive profitabilities resembles Bernanke's Bad News Principle, though in a weaker form.

The following Corollary to Proposition 3 shows how the presence of financial constraints affects marginal $q$ and the optimal investment-capital ratio.

Corollary 1 If $x^{*}>0$, then for Regime $j \in\{H, L\}$ and $0 \leq x \leq x^{*}$,

1. $q_{M}^{j}(x)<\widetilde{v}^{j}$, where $\widetilde{v}^{j}$ is the value of $q$ (both marginal and average) in the absence of financial constraints.

2. $i^{j}(x)<\widetilde{i}^{j}$, where $\widetilde{i}^{j}$ is the optimal value of the investment-capital ratio in the absence of financial constraints.

Statement 2 in Corollary 1 highlights the impact of financial constraints on optimal investment. Specifically, in both regimes, financial constraints reduce the optimal value of the investment-capital ratio relative to the optimal value of the investment-capital ratio in the absence of financial constraints, regardless of whether the financial constraints are currently binding. The channel by which financial constraints reduce optimal investment is through a reduction in marginal $q$. The relationship between the optimal investmentcapital ratio and marginal $q$ is not affected by the introduction of financial constraints, consistent with Chirinko (1993) who states "Even though financial market frictions impinge on the firm, $q$ is a forward-looking variable capturing the ramifications of these constraints on all the firm's decisions." (p. 1903) Chirinko's statement does not specify whether $q$ is average $q$ or marginal $q$. If we interpret Chirinko's statment to be about marginal $q$, then his statement applies to our analysis. However, marginal $q$ is not directly observable, so empirical analyses typically use average $q$ as a proxy for marginal $q$. As we show in Section 4, financial constraints drive a wedge between average $q$ and marginal $q$, and thereby affect the relationship between the investment-capital ratio and average $q$.

\section{Average $q$ vs. Marginal $q$}

With quadratic adjustment costs, the optimal investment-capital ratio, $i$, is a linear function of marginal $q$, as shown in equation (23). A challenge for empirical analysis is that marginal $q$ is not directly observable. However, average $q$, which is the value of the firm divided by its capital stock, is observable. In the absence of financing constraints, average $q$ and marginal 
$q$ are equal to each other under the Hayashi (1982) conditions that profits are proportional to the capital stock and the adjustment cost function is linearly homogeneous in investment and the capital stock. Empirical studies typically appeal to the Hayashi conditions to justify using average $q$ as a proxy for marginal $q$ in investment regressions. However, financing constraints drive a wedge between average $q$ and marginal $q$ even if the Hayashi conditions hold. Therefore, using average $q$ as a proxy for marginal $q$ in an investment regression introduces a form of measurement error in the regressor. Unlike classical measurement error, which biases an estimated coefficient toward zero, the measurement error in average $q$ is non-classical and, as we will show, biases the estimated coefficient on average $q$ upward.

In computing average $q$, empirical studies typically adjust the value of the firm by subtracting the value of liquid assets from the overall value of the firm. In our model, liquid assets are cash on hand, $X$, so the adjusted value of the firm in Regime $j$ is $V^{j}(K, X)-X$. The replacement cost of the capital stock is typically the cost of purchasing capital, not including adjustment costs, which is simply $K$ in our model. Therefore, average $q$ in Regime $j$ is

$$
q_{A}^{j}(x) \equiv \frac{V^{j}(K, X)-X}{K}=v^{j}(x)-x, \text { for } j \in\{H, L\} .
$$

Subtract the value of marginal $q$ in equation (24) from the value of average $q$ in equation (31) to obtain

$$
q_{A}^{j}(x)-q_{M}^{j}(x)=\left(1-\frac{1}{v_{x}^{j}(x)}\right) v^{j}(x) \geq 0, \quad \text { for } 0 \leq x \leq x^{*},
$$

where the inequality follows from $v^{j}(x) \geq 0$ and $v_{x}^{j}(x) \geq 1$. Therefore, average $q$ is greater than or equal to marginal $q$.

The boundary condition $v_{x}^{H}\left(x^{*}\right)=1$ in equation (18) implies

$$
q_{M}^{H}\left(x^{*}\right)=v^{H}\left(x^{*}\right)-x^{*}=q_{A}^{H}\left(x^{*}\right),
$$

so average $q$ and marginal $q$ are equal to each other in Regime $H$ when $x=x^{*}$.

In the case in which the salvage value of the firm is zero, i.e., $\alpha=0$, the boundary condition $v^{L}(0)=\alpha$ in equation (20) implies

$$
q_{A}^{L}(0)=0=q_{M}^{L}(0), \text { when } \alpha=0
$$

so average $q$ and marginal $q$ are equal to each other in Regime $L$ when $x=0$ and $\alpha=0$. Except for the two cases in equations (33) and (34), average $q$ is strictly greater than marginal $q$. 


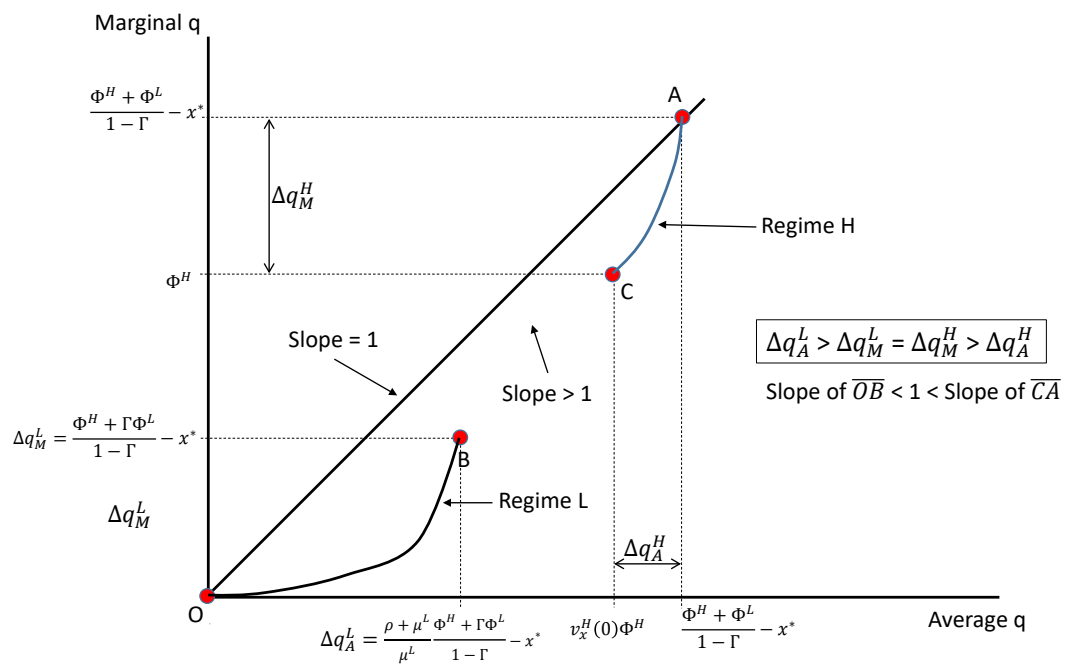

Figure 1: Marginal and Average q when salvage value, $\alpha$, equals zero.

Figure 1 illustrates the relationship between average $q$ on the horizontal axis and marginal $q$ on the vertical axis for the case in which the salvage value $\alpha$ equals zero. In this case, there is a closed-form solution for $x^{*}$, as shown in equation (B.7) in Appendix B. (We analyze the impact of positive $\alpha$ in Section 5.) Underlying this figure is the assumption that $\Phi^{L}+\Gamma \Phi^{H}>0$, which is equivalent to $x^{*}>0$ and $v_{x}^{H}(0)>1$. The origin, Point $\mathrm{O}$, represents equation (34) in Regime $L$ when the firm has no cash on hand. In this case, the value of the firm is zero, so average $q$ and marginal $q$ are both zero. The curve connecting Point $\mathrm{O}$ and Point $\mathrm{B}$ represents average $q$ and marginal $q$ in Regime $L$ for values of $x$ up to and including $x=x^{*}$. At the origin, where $x=0, \frac{d q_{M}^{L}}{d x}=0$ and $\frac{d q_{A}^{L}}{d x}>0$ so the slope of the curve is zero at the origin. ${ }^{16}$ For $x>0$, both average $q$ and marginal $q$ increase as $x$ increases, so this curve slopes upward, reaching its maximum at Point B where $x=x^{*}$. Importantly, except at the origin, this curve lies everywhere below the solid line through the origin with slope equal to one.

The values of average $q$ and marginal $q$ in Regime $H$ are shown along the curve connecting Point $\mathrm{C}$ and Point A. Again, since both average $q$ and marginal $q$ increase as $x$ increases,

${ }^{16}$ Differentiating equation (31) with respect to $x$ yields $\frac{d q_{A}^{j}(x)}{d x}=v_{x}^{j}(x)-1 \geq 0$, with strict inequality everywhere except in Regime $H$ at $x=x^{*}$. Differentiating equation (24) with respect to $x$ yields $\frac{d q_{M}^{j}(x)}{d x}=$ $-\frac{v^{j}(x)}{\left[v_{x}^{j}(x)\right]^{2}} v_{x x}^{j}(x) \geq 0$, with strict inequality everywhere except in Regime $L$ when $x=0\left(\right.$ where $v^{L}(0)=\alpha=$ 0 ) and Regime $H$ when $x=x^{*}$ (where $\left.v_{x x}^{H}\left(x^{*}\right)=0\right)$. 


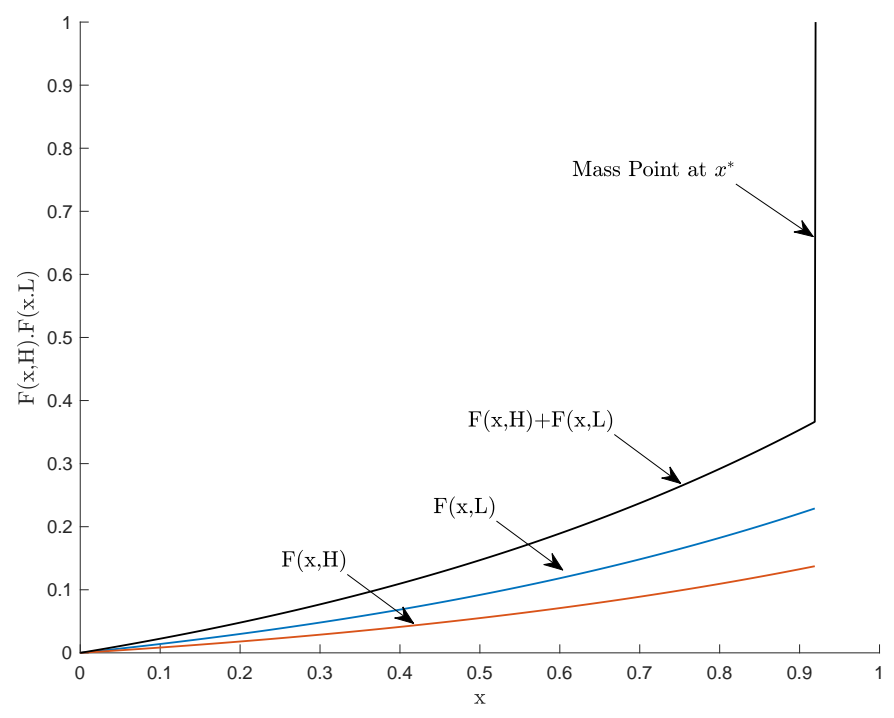

Figure 2: An illustration of $F(x, H), F(x, L)$.

the slope of this curve is positive for all $x$. As $x$ gets arbitrarily close to $x^{*}$, that is, in the neighborhood of Point $A$, the curve becomes vertical. ${ }^{17}$ Equation (33) states that Point A lies on the line through the origin with slope equal to one. However, all other points on this curve lie below the this line.

\subsection{The Distribution of Cash on Hand}

Continue to assume that $\Phi^{L}+\Gamma \Phi^{H}>0$ so that $x^{*}>0$. Consider a set of sample paths generated by optimal payout and investment behavior. Specifically, each sample path is generated by a firm that begins operation in Regime $H$ with $x=0$ and follows optimal investment and payout policy. Eventually, the firm will terminate in some Regime $L$ when $x=0$. Along any such realized continuous-time path of $x_{t}, x_{t}$ equals zero at precisely two points in time - when the firm is born and when the firm dies. The distribution of the continuous variable $x$ in Regime $H$ has a discrete mass at $x=x^{*}$ because once $x$ reaches $x^{*}$ in

\footnotetext{
${ }^{17}$ Evaluate the derivatives in footnote 16 at $x=x^{*}$, and use the boundary conditions in equations (18) and (19), to obtain $\frac{d q_{A}^{H}\left(x^{*}\right)}{d x}=v_{x}^{H}\left(x^{*}\right)-1=0$ and $\frac{d q_{M}^{H}\left(x^{*}\right)}{d x}=-\frac{v^{H}\left(x^{*}\right)}{\left[v_{x}^{H}\left(x^{*}\right)\right]^{2}} v_{x x}^{H}\left(x^{*}\right)=0$. Therefore, to calculate the slope $\left.\frac{d q_{M}^{H}(x)}{d q_{A}^{H}(x)}\right|_{x=x^{*}}=\frac{d q_{M}^{H}\left(x^{*}\right)}{d x} / \frac{d q_{M}^{H}\left(x^{*}\right)}{d x}$ requires L'Hopital's Rule. Calculating derivatives of the numerator and denominator of $\frac{d q_{M}^{H}(x)}{d q_{A}^{H}(x)}$ and evaluating them at $x=x^{*}$ yields $\left.\frac{d^{2} q_{M}^{H}(x)}{d x^{2}}\right|_{x=x^{*}}=-\frac{v^{H}\left(x^{*}\right)}{\left[v_{x}^{H}\left(x^{*}\right)\right]^{2}} v_{x x x}^{H}(x)<0$ and $\frac{d^{2} q_{A}^{H}\left(x^{*}\right)}{d x^{2}}=v_{x x}^{H}\left(x^{*}\right)=0$, so $\frac{d q_{M}^{H}(x)}{d q_{A}^{H}(x)}$ approaches infinity as $x$ approaches $x^{*}$.
} 
Regime $H, x$ remains equal to $x^{*}$ for the remainder of that regime. Though the distribution of $x$ in Regime $L$ does not have a mass at $x=x^{*}$, much of the mass of that distribution is near $x=x^{*}$ because there will be many transitions from Regime $H$ into Regime $L$ when $x=x^{*}$.

To describe the distribution of $x$ more fully, let $F(x, j)$ be the probability, in a large set of sample paths, of the joint event that Regime $j$ prevails and cash on hand, $x_{t}<x$. Appendix $\mathrm{C}$ derives

$$
F(x, j)=\frac{1}{\left|\phi^{(j)}\right|} a\left(e^{b x}-1\right) \text { for } j \in\{H, L\} \text { and } 0 \leq x \leq x^{*},
$$

where $a>0$ and $b>0$ are defined in equations (C.46) and (C.36), respectively. The distribution of $x$ has a mass equal to $\frac{1}{\mu^{L}} a b e^{b x^{*}}>0$ at $x=x^{*}$ in Regime $H$. The regime-specific distribution functions are proportional to each other. Specifically, $F(x, H)=\frac{-\phi^{L}}{\phi^{H}} F(x, L)$. The density is $F_{x}(x, j)=\frac{a b}{\left|\phi^{(j)}\right|} e^{b x}$. Figure 2 illustrates the functions $F(x, L)$ and $F(x, H)$ for the following configuration of parameters: $\alpha=0, \rho=0.04, \phi^{H}=0.25, \phi^{L}=-0.15$, $\mu^{H}=0.25$, and $\mu^{L}=0.10$. With these parameter values, $x^{*}=0.92$.

\subsection{Regressions on Average $q$}

In this section we analyze various regressions in which average $q$ is a regressor, either by itself or along with cash flow as an additional regressor. We consider three scenarios of regressions that are defined by the sample of observations (either Regimes $H$ and $L$ or Regime $L$ only) and the set of regressors (either average $q$ only or both average $q$ and cash flow). In order to distinguish the coefficients in the various regressions, Table 4.2 presents the notation for the various regression coefficients in the three scenarios. In both Scenarios I and II, the sample consists of both Regime $H$ and Regime $L$. Scenarios I and II are distinguished from each other by the number of regressors: in Scenario I, average $q$ is the lone regressor, and in Scenario II both average $q$ and cash flow are regressors. Scenario III is distinguished by its sample, which consists only of observations in Regime $L .{ }^{18}$

Within each of the three scenarios, there are two regressions: one regression uses marginal $q$ as the dependent variable and the other regression uses the investment-capital ratio $i$ as the dependent variable. Within each scenario, the regression coefficients (except the intercept) in the regression with $i$ as the dependent variable are $\frac{1}{\theta}$ times the corresponding coefficients

\footnotetext{
${ }^{18}$ Cash flow, $\phi$, cannot be a regressor when the sample is limited to a single regime because there is no within-regime variation in $\phi$.
} 


\begin{tabular}{cccc}
\hline & Regressions with Marginal $q$ as Dependent Variable & \\
\hline Scenario & Sample & Regressor $(\mathbf{s})$ & Coefficients \\
\hline$I$ & Regimes $H$ and $L$ & $q_{A}$ & $\beta_{q}^{I}$ \\
$I I$ & Regimes $H$ and $L$ & $q_{A}$ and $\phi$ & $\beta_{q}^{I I}$ and $\beta_{\phi}$ \\
$I I I$ & Regime $L$ & $q_{A}$ & $\beta_{q}^{I I I}$ \\
\hline
\end{tabular}

Table 1: Three different regression scenarios

in the regression with marginal $q$ as the dependent variable because the optimal investmentcapital ratio $i$ is a deterministic linear function of marginal $q$ with slope $\frac{1}{\theta}$. The regressions with marginal $q$ as the dependent variable are necessarily theoretical regressions because marginal $q$ is not directly observable when $v_{x}^{j}(x)>1$. However, these theoretical regressions are useful to examine because the magnitudes of the regression coefficients do not depend on $\theta$, provided that $\theta$ is sufficiently large. We begin by analyzing regressions with marginal $q$ as the dependent value in all three scenarios and then we examine regressions with the investment-capital ratio as the dependent variable.

\subsubsection{Regressions of Marginal $q$ on Average $q$}

Before analyzing regressions of the investment-capital ratio on average $q$, we start by examining theoretical regressions of marginal $q$ on average $q$. Since financing costs drive a wedge between average $q$ and marginal $q$, the regression coefficient can differ from one, even when the Hayashi conditions hold.

Figure 3 uses the density function of Figure 2 to illustrate the joint distribution of marginal $q$ and average $q$ in the same two-dimensional diagram as in Figure 1 . The thickness of the curve $\mathrm{OB}$ increases from the origin $\mathrm{O}$ to Point $\mathrm{B}$, indicating the increasing density of observations in Regime $L$ as $x$ increases from 0 at the origin to $x^{*}$ at Point B. Similarly, the curve extending from Point $\mathrm{C}$ to Point $\mathrm{A}$ represents observations in Regime $H$, with increasing density as $x$ increases from 0 at Point $\mathrm{C}$ to $x^{*}$ at Point $\mathrm{A}$. In addition, there is a mass at Point A.

Now consider the population regression of marginal $q$ on average $q$ using the full sample of observations from both Regime $L$ and Regime $H$ (Scenario I). As indicated in Table 4.2, $\beta_{q}^{I}$ is the population regression coefficient on average $q$. The fitted line of this regression is the steeply upward sloping line that passes a bit below Point $\mathrm{A}$ in the upper right portion of Figure 3. Since there is a mass of observations at Point A, and the density $F_{x}(x, H)$ 


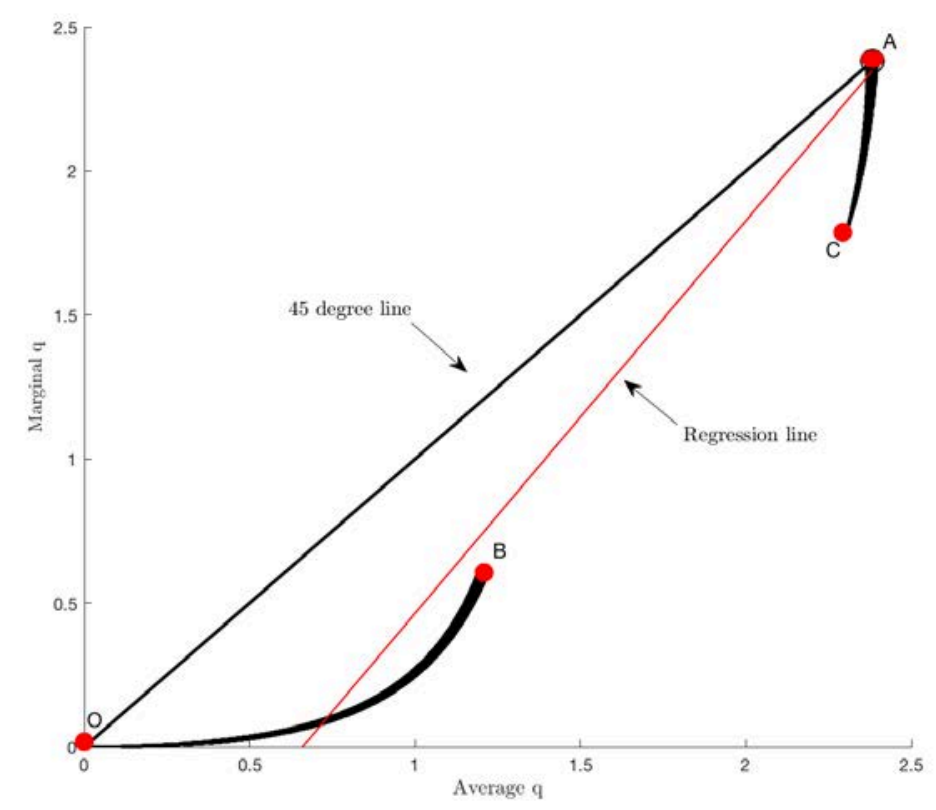

Figure 3: Regression of Marginal q on Average q.

$=\frac{a b}{\left|\phi^{(j)}\right|} e^{b x}$ is increasing in $x$, much of the mass of observations in Regime $H$ is located at or near Point A. Also, in Regime $L$, much of the mass of the distribution is near Point B (though there is not a mass at Point B). Thus, most of the mass of the points in Figure 3 is located near Points A and B. Therefore, the fitted line passes close to these points, as shown by the regression line in Figure 3. The slope of this fitted line, $\beta_{q}^{I}$, exceeds one because Point A pulls the regression line upward toward the 45-degree line for high values of average $q$ and Point B pulls the regression line away from the 45-degree line for low values of average $q$. In this specific example, the slope is $\beta_{q}^{I}=1.33$. Empirical analyses of investment and $q$ typically appeal to the Hayashi conditions to use average $q$ as a proxy for marginal $q$. Under the Hayashi conditions, and in the absence of financial constraints, any difference between average $q$ and marginal $q$ is regarded as measurement error in average $q$. If the measurement error is classical measurement error - that is, has zero mean and is uncorrelated with marginal $q$-then a regression of marginal $q$ on average $q$ would have a positive slope smaller than one. Remarkably, we have shown that in the presence of financial constraints, the slope of the fitted line from the regression of marginal $q$ on average $q$ is greater than one. Evidently, the measurement error associated with using average $q$ as a proxy for marginal $q$ in the presence of financial constraints is not classical measurement error. ${ }^{19}$

\footnotetext{
${ }^{19}$ Equation (32) provides an expression for this measurement error. Not only does this measurement error
} 
In the context of the model presented here, there is a simple way to create a sample of observations that is free of non-classical measurement error in average $q$ induced by financial constraints. A sample that includes only observations at times when optimal dividends are positive will not contain this measurement error. In the model presented here, the firm will pay dividends only if the marginal value of cash on hand, $v_{x}^{j}(x)$, equals one. Substituting $v_{x}^{j}(x)=1$ into the expression for measurement error in equation (32) reveals that the measurement error in average $q$ is zero when $v_{x}^{j}(x)=1$. In this model, the firm pays dividends only in Regime $H$ and only at a single value of $x$, namely, $x^{*}$. Because the distribution of $x$ has a potentially large mass at $x=x^{*}$ in Regime $H$, a substantial fraction of any set of observations on investment and average $q$ will be associated with positive dividends.

If one constructs a sample from a set of ex ante identical firms (that is, firms with identical values of $\alpha, \rho, \phi^{H}, \phi^{L}, \mu^{H}$, and $\mu^{L}$, but idiosyncratic realizations of regimes) and includes only observations for which dividends are positive, then all observations in the sample have the same value of average $q$. In such a sample, all observations have average $q$ equal to $q_{A}^{H}\left(x^{*}\right)$, so there is no variation in the independent variable in a regression of marginal $q$ on average $q$. To get variation in the independent variable, we could generate observations from sample paths of different firms with different values of the parameters ${ }^{20}$. Alternatively, one might consider one or more ex ante identical firms that face multiple profitability regimes and pay dividends in different regimes at different values of average $q$ in each regime. While the quantitative methods of this paper could be extended to analyze such a model, we do not pursue this extension in the current paper.

\subsubsection{Regressions of Marginal $q$ on Average $q$ and Cash Flow}

Now consider the population regression of marginal $q$ on average $q$ and cash flow $\phi$ (Scenario II). The impact of adding cash flow as a second regressor is that Points A and B continue to assert a strong influence on the fitted line, but do so without steepening the fitted line. In a two-regime stochastic framework, there are only two values of cash flow, $\phi^{H}>0$ and $\phi^{L}<0$, so cash flow is simply a binary variable that indicates the regime. Figure 4 exploits the fact that cash flow is a binary variable to represent the 3-dimensional relationship among

have a positive mean, it is not independent of marginal $q$.

${ }^{20}$ If the dependent variable is the investment-capital ratio $i$ rather than marginal $q$, then the firms must have identical values of $\theta$ so that the slope of the population regression of $i$ on average $q$ will equal $\frac{1}{\theta}$ if all of the observations have positive dividends. 


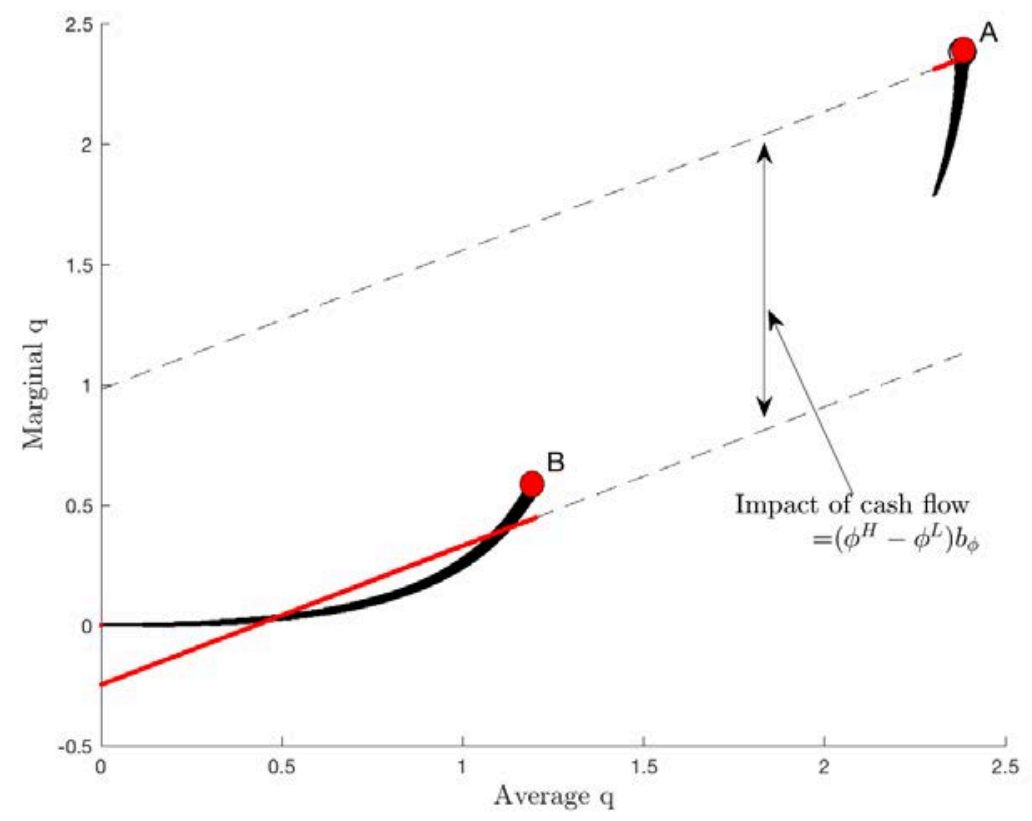

Figure 4: Regression of Marginal q on Average q and Cash Flow.

marginal $q$, average $q$, and cash flow in a 2-dimensional diagram. The common slope of the two parallel dashed lines equals $\beta_{q}^{I I}$, the regression coefficient on average $q$ in this regression. The solid segment of the lower of the two lines is the fitted line of the regression for observations in Regime $L$, when cash flow is $\phi^{L}$. The short solid segment of the higher of the two lines is the fitted line of the regression for observations in Regime $H$, when cash flow is $\phi^{H}$. The impact of cash flow is shown in Figure 4 as the vertical distance between the two upward-sloping dashed lines, which is $\left(\phi^{H}-\phi^{L}\right) \beta_{\phi}>0$, where $\beta_{\phi}>0$ is the coefficient on cash flow in a full-sample regression of marginal $q$ on average $q$ and cash flow $\phi$.

The distribution function in Figure 2 shows that most of the observations in Regime $H$ are located at or near Point A, which is a mass point. Figure 4 shows that within Regime $H$ there is very little variation in average $q$ compared to the variation in average $q$ in Regime $L$. With so little variation in average $q$ in Regime $H$, the within-regime variation in average $q$ has very little impact on the estimated coefficient of average $q$. To exaggerate for the sake of expositional clarity, OLS recognizes that variation in average $q$ within Regime $H$ is nearly worthless in estimating the coefficient on average $q$ in a multiple regression; therefore, OLS essentially treats all observations in Regime $H$ as being located at Point A.

The estimated coefficients in the multiple regression on average $q$ and cash flow (Scenario 


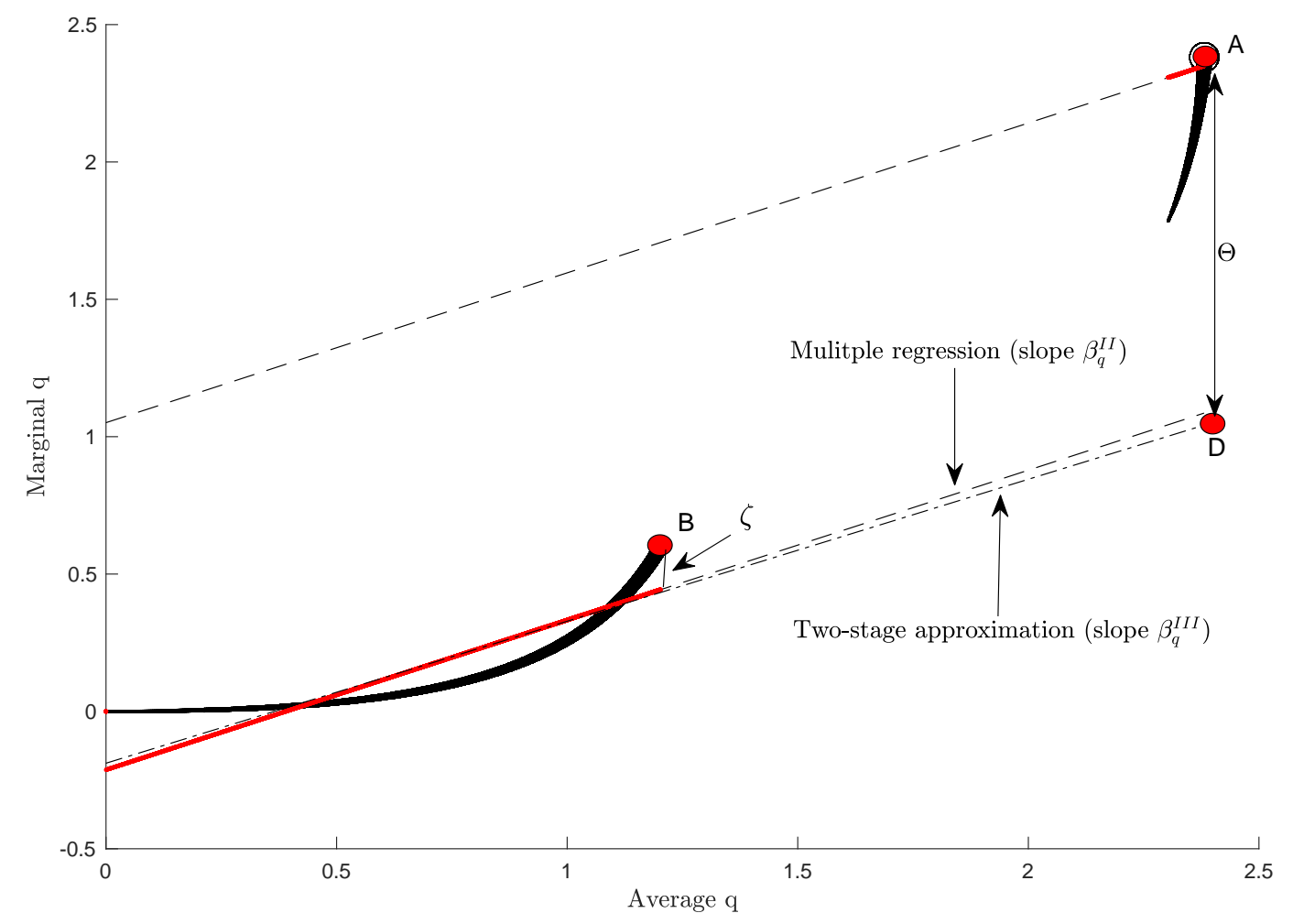

Figure 5: Regression of Marginal q on Average q and Cash Flow and approximate two-step procedure.

II) can be approximated by a 2 -stage procedure. Stage 1 provides an alternative estimate of the coefficient on average $q$ using a univariate regression in Regime $L$ (Scenario III) and Stage 2 provides an alternative calculation of the coefficient on cash flow.

Stage 1: Using only the observations in Regime $L$, run a univariate regression of marginal $q$ on average $q$ (Scenario III) to obtain the coefficient on average $q$, denoted $\beta_{q}^{I I I}$, and the constant, $\beta_{0}$. Define and calculate

$$
\zeta \equiv q_{M}^{L}\left(x^{*}\right)-\left[\beta_{0}+\beta_{q}^{I I I} q_{A}^{L}\left(x^{*}\right)\right],
$$

which is the value of the approximation error when average $q$ equals $q_{A}^{L}\left(x^{*}\right)$.

Stage 2: Find the point (call it Point D) on the fitted regression line from Stage 1 that is vertically below Point A. Calculate $\Theta$, which is the distance from point D to point A, and equate $\Theta$ with the impact of cash flow $\left(\phi^{H}-\phi^{L}\right) \widehat{\beta}_{\phi}$, to obtain an alternative measure 


\begin{tabular}{lcc}
\hline Scenario & coefficent on average $q$ & cash flow coefficient \\
\hline I & $\beta_{q}^{I}=1.33$ & --- \\
II & $\beta_{q}^{I I}=0.55$ & $\beta_{\phi}=3.16$ \\
III & $\beta_{q}^{I I I}=0.52$ & $\widehat{\beta}_{\phi}=3.35$ \\
\hline
\end{tabular}

Table 2: A summary of the regression coefficients

of the coefficient on cash flow as

$$
\widehat{\beta}_{\phi}=\frac{\Theta}{\phi^{H}-\phi^{L}} \text {. }
$$

Appendix D presents the details of the implementation of this 2-step procedure for the parameter values underling the quantitative example in Figure 4. In the final row of Table 2, labelled Scenario III, are the values of $\beta_{q}^{I I I}$, the coefficient on average $q$ obtained in Stage 1 , and the value of $\widehat{\beta}_{\phi}$, the coefficient on cash flow deduced in Stage 2. These coefficients in Scenario III are each about $5 \%$ different than the coefficients in the full-sample regression, Scenario II, of the investment-capital ratio on average $q$ and cash flow. Figure 5 illustrates how well the 2 -stage procedure approximates the multiple regression of marginal $q$ on average $q$ and cash flow.

\subsubsection{Investment Regressions}

Now consider regressions in which the investment-capital ratio, $i$, is the dependent variable. As discussed earlier, replacing marginal $q$ with $i$ as the dependent variable in the regressions in Scenarios I, II, and III simply multiplies all of the regression coefficients (except for the intercept) by $\frac{1}{\theta}$.

First consider the investment version of Scenario I, which is a univariate regression of the investment-capital ratio (rather than marginal $q$ ) on average $q$. The regression coefficient is $\frac{1}{\theta} \beta_{q}^{I}>\frac{1}{\theta}$, where the inequality follows from our finding that non-classical measurement error in average $q$ causes $\beta_{q}^{I}$ to exceed one. Therefore, an OLS regression of the investmentcapital ratio on average $q$ is likely to overstate $\frac{1}{\theta}$, equivalently, understate the adjustment cost parameter $\theta$. This finding is the opposite of the typical argument, which says that because of classical measurement error, the estimated coefficient $\frac{1}{\theta} \beta_{q}^{I}$ is likely to understate $\frac{1}{\theta}$ and hence overstate the adjustment cost parameter $\theta$. Our finding implies that measurement error arising from financial constraints cannot be the explanation for estimates of $\frac{1}{\theta} \beta_{q}^{I}$ that are typically regarded as too low. 
The conventional wisdom is that in regressions of the investment-capital ratio on average $q$ and cash flow (Scenario II), the coefficient on average $q$ measures the sensitivity of the investment-capital ratio to the expectations of future marginal profits of capital and the coefficient on cash flow reflects the impact of financing constraints on investment. The two-stage procedure discussed above leads to a different interpretation. To analyze the interpretation suggested by the 2-stage procedure, consider the impact on the investmentcapital ratio of a switch from Regime $L$ to Regime $H$, when $x=x^{*}$. First, we analyze this impact, which is $i^{H}\left(x^{*}\right)-i^{L}\left(x^{*}\right)$, within our model and then we analyze the impact on $i^{H}\left(x^{*}\right)-i^{L}\left(x^{*}\right)$ calculated from the coefficients of the 2-stage procedure.

To calculate the impact within our model, use $i^{H}\left(x^{*}\right)-i^{L}\left(x^{*}\right)=\frac{1}{\theta}\left(q_{M}^{H}\left(x^{*}\right)-q_{M}^{L}\left(x^{*}\right)\right)$ and the definitions of average $q$ and marginal $q$ to obtain ${ }^{21}$

$$
i^{H}\left(x^{*}\right)-i^{L}\left(x^{*}\right)=\underbrace{\frac{1}{\theta}\left[q_{A}^{H}\left(x^{*}\right)-q_{A}^{L}\left(x^{*}\right)\right]}_{\text {impact of expected future profitability }}+\underbrace{\frac{1}{\theta}\left[v_{x}^{L}\left(x^{*}\right)-1\right] \frac{v^{L}\left(x^{*}\right)}{v_{x}^{L}\left(x^{*}\right)}}_{\text {scaled shadow price of constraint }} .
$$

The right hand side of equation (38) expresses the difference in the investment-capital ratio when $x=x^{*}$ in the two regimes as the sum of two components. The first component equals the difference in average $q$ when $x=x^{*}$ in the two regimes-which is equal to the difference in valuation, $v^{H}\left(x^{*}\right)-v^{L}\left(x^{*}\right)$-multiplied by $\frac{1}{\theta}$. This component, which is proportional to the difference in valuation across the two regimes, captures the response of the investment-capital ratio to the change in the present value of future profitability when the regime switches to Regime $H$ from Regime $L .^{22}$ The second component is the product of $(1) \frac{1}{\theta}$, (2) $v_{x}^{L}\left(x^{*}\right)-1$, which is the shadow price of the constraint $x \geq 0$, and $(3) \frac{v^{L}\left(x^{*}\right)}{v_{x}^{L}\left(x^{*}\right)}$, which is the value of the firm in Regime $L$ when $x=x^{*}$, normalized by the marginal valuation of cash on hand, $v_{x}^{L}\left(x^{*}\right)$. This component reflects the impact of the financial constraint $x \geq 0$.

${ }^{21}$ The definition of average $q, q_{A}^{j}(x) \equiv v^{j}(x)-x$, implies $q_{A}^{H}(x)-q_{A}^{L}(x)=v^{H}(x)-v^{L}(x)$. The definition of marginal $q, q_{M}^{j}(x) \equiv \frac{v^{j}(x)}{v_{x}^{j}(x)}-x$, along with $v_{x}^{H}\left(x^{*}\right)=1$ implies $q_{M}^{H}\left(x^{*}\right)-q_{M}^{L}\left(x^{*}\right)=v^{H}\left(x^{*}\right)-\frac{v^{L}\left(x^{*}\right)}{v_{x}^{L}\left(x^{*}\right)}$. Therefore, $q_{M}^{H}\left(x^{*}\right)-q_{M}^{L}\left(x^{*}\right)=\left[v^{H}\left(x^{*}\right)-v^{L}\left(x^{*}\right)\right]+v^{L}\left(x^{*}\right)-\frac{v^{L}\left(x^{*}\right)}{v_{x}^{L}\left(x^{*}\right)}=\left[q_{A}^{H}(x)-q_{A}^{L}(x)\right]+\left[v_{x}^{L}\left(x^{*}\right)-1\right] \frac{v^{L}\left(x^{*}\right)}{v_{x}^{L}\left(x^{*}\right)}$.

${ }^{22}$ Since a regime is defined by the value of cash flow, $\phi$, there is no within-regime variation in $\phi$. Therefore, all of the within-regime variation in average $q$ arises from variation in cash on hand $x$, which affects the expected present of cash flows over the lifetime of the firm by affecting the expected lifetime of the firm. For instance, an increase in $x$ allows the firm to live a longer period of time before it is forced to terminate in some Regime $L$ when it runs out of cash. This increase in the firm's projected longevity increases the value of the firm $v^{j}(x)$ and increases $q_{A}^{j}(x)=v^{j}(x)-x$ since $v_{x}^{j}(x)>1$ for $0 \leq x<x^{*}$. Thus, $\beta_{q}^{I I I}$ reflects the variation in average $q$ that arises from within-Regime $L$ variation in cash on hand $x$, but it does not reflect the variation in average $q$ that arises from a change in regime. Nevetheless, as we show, the value of $\beta_{q}^{I I I}$ is close to $\beta_{q}^{I I}$. 
Now consider the impact on $i^{H}\left(x^{*}\right)-i^{L}\left(x^{*}\right)$ implied by the 2 -stage procedure. As shown in Appendix D

$$
i^{H}\left(x^{*}\right)-i^{L}\left(x^{*}\right)=\underbrace{\beta_{q}^{I I I} \frac{1}{\theta}\left[q_{A}^{H}\left(x^{*}\right)-q_{A}^{L}\left(x^{*}\right)\right]}_{\text {regression impact of average } q}+\underbrace{\left(\phi^{H}-\phi^{L}\right) \frac{1}{\theta} \widehat{\beta}_{\phi}}_{\text {regression impact of cash flow }}-\frac{1}{\theta} \zeta .
$$

The sum of the first two terms on the right hand side of equation (39) is the fitted value of $i^{H}\left(x^{*}\right)$ minus the fitted value of $i^{L}\left(x^{*}\right)$, where the fitted values in Regime $j$ are calculated as a constant plus $\frac{1}{\theta} \beta_{q}^{I I I} q_{A}^{j}\left(x^{*}\right)+\frac{1}{\theta} \widehat{\beta}_{\phi} \phi^{j}$, and the coefficients $\beta_{q}^{I I I}$ and $\widehat{\beta}_{\phi}$ are obtained from the 2-stage procedure. The third term in equation (39) is proportional to the approximation error $\zeta$ defined in equation $(36)$.

To compare the impacts of expected future profitability and the scaled impact of the financial constraint within the model in equation (38) with the regression impacts of average $q$ and cash flow in the 2 -stage procedure as shown in equation (39), rewrite equation (38) as

$$
\begin{aligned}
i^{H}\left(x^{*}\right)-i^{L}\left(x^{*}\right)= & \underbrace{\beta_{q}^{I I I} \frac{1}{\theta}\left[q_{A}^{H}\left(x^{*}\right)-q_{A}^{L}\left(x^{*}\right)\right]}_{\text {Term } 1}+\underbrace{\left(1-\beta_{q}^{I I I}\right) \frac{1}{\theta}\left[q_{A}^{H}\left(x^{*}\right)-q_{A}^{L}\left(x^{*}\right)\right]}_{\text {Term } 2} \\
& +\underbrace{\frac{1}{\theta}\left[v_{x}^{L}\left(x^{*}\right)-1\right] \frac{v^{L}\left(x^{*}\right)}{v_{x}^{L}\left(x^{*}\right)}}_{\text {Term } 3}
\end{aligned}
$$

Terms 1 and 2 in equation (40) simply split the first term in equation (38), which is the impact of expected future profitability, into two components, and Term 3 is the impact of the scaled shadow price of the constraint, which is the second term in equation (38). The mapping between equations (38) and (40) is shown by the first two columns of Table 3 . The regression impacts of average $q$ and cash flow, based on equation (39), are shown in the third column of Table 3 .

The regression impact of average $q$ is simply equal to Term 1 in equation (40). The regression impact of cash flow in equation (39) is approximately equal to the sum of Term 2 and Term 3 in equation (40). ${ }^{23}$ Finally, a comparison of column 1 and column 3 in Table 3 shows that the regression impact of average $q$ in column 3 captures only a fraction $\beta_{q}^{I I I}$ of the impact of expected future profitability in column 1. Also the regression impact of cash

\footnotetext{
${ }^{23}$ The regression impact of cash flow in equation (39) differs from the sum of Term 2 and Term 3 in equation (40) by the approximation error $\zeta$ multiplied by $\frac{1}{\theta}$.
} 


\begin{tabular}{|c|c|c|}
\hline Model & TERMS 1,2 , and 3 & 2-stage Regression \\
\hline \multirow{2}{*}{$\begin{array}{l}\text { Impact of expected } \\
\text { future profitability } \\
\frac{1}{\theta}\left[q_{A}^{H}\left(x^{*}\right)-q_{A}^{L}\left(x^{*}\right)\right]\end{array}$} & $\begin{array}{c}\beta_{q}^{I I I} \frac{1}{\theta}\left[q_{A}^{H}\left(x^{*}\right)-q_{A}^{L}\left(x^{*}\right)\right] \\
(\text { TERM 1) }\end{array}$ & $\begin{array}{l}\text { Regression impact } \\
\text { of average } q \\
\beta_{q}^{I I I} \frac{1}{\theta}\left[q_{A}^{H}\left(x^{*}\right)-q_{A}^{L}\left(x^{*}\right)\right]\end{array}$ \\
\hline & $\begin{array}{c}\left(1-\beta_{q}^{I I I}\right) \frac{1}{\theta}\left[q_{A}^{H}\left(x^{*}\right)-q_{A}^{L}\left(x^{*}\right)\right] \\
(\text { TERM 2) }\end{array}$ & \multirow{2}{*}{$\begin{array}{l}\text { Regression impact } \\
\text { of cash flow } \\
\qquad\left(\phi^{H}-\phi^{L}\right) \frac{1}{\theta} \widehat{\beta}_{\phi}\end{array}$} \\
\hline \multirow[t]{2}{*}{$\begin{array}{l}\text { Scaled shadow price } \\
\text { of financial constraint } \\
\frac{1}{\theta}\left[v_{x}^{L}\left(x^{*}\right)-1\right] \frac{v^{L}\left(x^{*}\right)}{v_{x}^{L}\left(x^{*}\right)}\end{array}$} & \multirow[t]{2}{*}{$\begin{array}{c}\frac{1}{\theta}\left[v_{x}^{L}\left(x^{*}\right)-1\right] \frac{v^{L}\left(x^{*}\right)}{v_{x}^{L}\left(x^{*}\right)} \\
(\text { TERM 3) }\end{array}$} & \\
\hline & & Approximation error $\frac{1}{\theta} \zeta$ \\
\hline
\end{tabular}

Table 3: Impacts of expected future profitability and shadow price of the financial constraint in the model and regression impacts of average $\mathrm{q}$ and cash flow in investment regressions.

flow in column 3 combines the scaled shadow price of the financial constraint and a fraction $1-\beta_{q}^{I I I}$ of the impact of expected future profitability in column 1.

\section{Impact of Salvage Value}

Proposition 3 implies that $v^{j}\left(x^{*}\right)$ and $q_{M}^{j}\left(x^{*}\right)$ are invariant to the salvage value $\alpha$ if $x^{*}>0$. This result may be surprising since the value of the firm when $x=x^{*}$ equals the sum of (1) cash on hand, $x^{*} K,(2)$ the expected present value of the stream of operating profits, net of investment costs, over the lifetime of the firm; and (3) the expected present value of the salvage value received upon termination of the firm. It might seem that an increase in the salvage value of capital would increase $v^{j}\left(x^{*}\right)$ and $q_{M}^{j}\left(x^{*}\right)$. In this section, we analyze the impact of salvage value and resolve what might seem like a contradiction.

In equations (B.1) and (B.2) in Appendix B, we present closed-form expressions for $v^{H}(x)$ 
and $v^{L}(x)$, given the optimal value of $x^{*}$, that can be rewritten, respectively, as

$$
v^{H}(x) \equiv g^{H}(u)
$$

where $u \equiv x-x^{*}$ and $g^{H}(u) \equiv c_{1} e^{\omega_{1} u}+c_{2} e^{\omega_{2} u}$ and

$$
v^{L}(x) \equiv g^{L}(u)
$$

where $g^{L}(u) \equiv \frac{\rho+\mu^{L}}{\mu^{L}}\left[c_{1}\left(1-\omega_{1} \Phi^{H}\right) e^{\omega_{1} u}+c_{2}\left(1-\omega_{2} \Phi^{H}\right) e^{\omega_{2} u}\right]$. Notably, the functions $g^{j}(u), j \in\{H, L\}$ are invariant to the salvage value $\alpha$. We exploit this invariance to prove Propositions 5 and 6 below.

Proposition 5 If $x^{*}>0$, then $\frac{d x^{*}}{d \alpha}=-\frac{1}{v_{x}^{L}(0)}<0$.

An increase in the salvage value $\alpha$ reduces $x^{*}$ because an increase in salvage value reduces the costliness of termination and thus reduces the incentive to hold cash on hand, $x$, as a means to forestall termination. Indeed, the consequent reduction in $x^{*}$ leads to an earlier termination of the firm along every realization path of profitability $\phi_{t} \cdot{ }^{24}$ Thus, for given values of the parameters $\phi^{H}, \phi^{L}, \mu^{H}$, and $\mu^{L}$ of the stochastic process for $\phi_{t}$, an increase in the salvage value $\alpha$ reduces the expected lifetime of the firm.

The definitions of $g^{H}(u)$ and $g^{L}(u)$ in equations (41) and (42), respectively imply

$$
v^{j}(x, \alpha) \equiv g^{j}\left(x-x^{*}(\alpha)\right), \quad j \in\{H, L\} .
$$

The following proposition exploits equation (43) to calculate the impacts of salvage value, $\alpha$, on $v^{j}(x), v_{x}^{j}(x)$ and $q_{M}^{j}(x)$.

Proposition 6 Define $q_{M}^{j}(x, \alpha) \equiv \frac{v^{j}(x, \alpha)}{v_{x}^{j}(x, \alpha)}-x$, for $j \in\{H, L\}$. Then for $0 \leq x \leq x^{*}$,
1. $v_{a}^{j}(x, \alpha)>0$
2. $v_{x a}^{j}(x, \alpha)<0$
3. $\frac{\partial q_{M}^{j}(x, \alpha)}{\partial \alpha}>0$.

\footnotetext{
${ }^{24}$ More precisely, along every realization path of $\phi_{t}$, a firm with a higher value of $\alpha$ will terminate earlier than or at the same time as an otherwise-identical firm with a lower value of $\alpha$.
} 


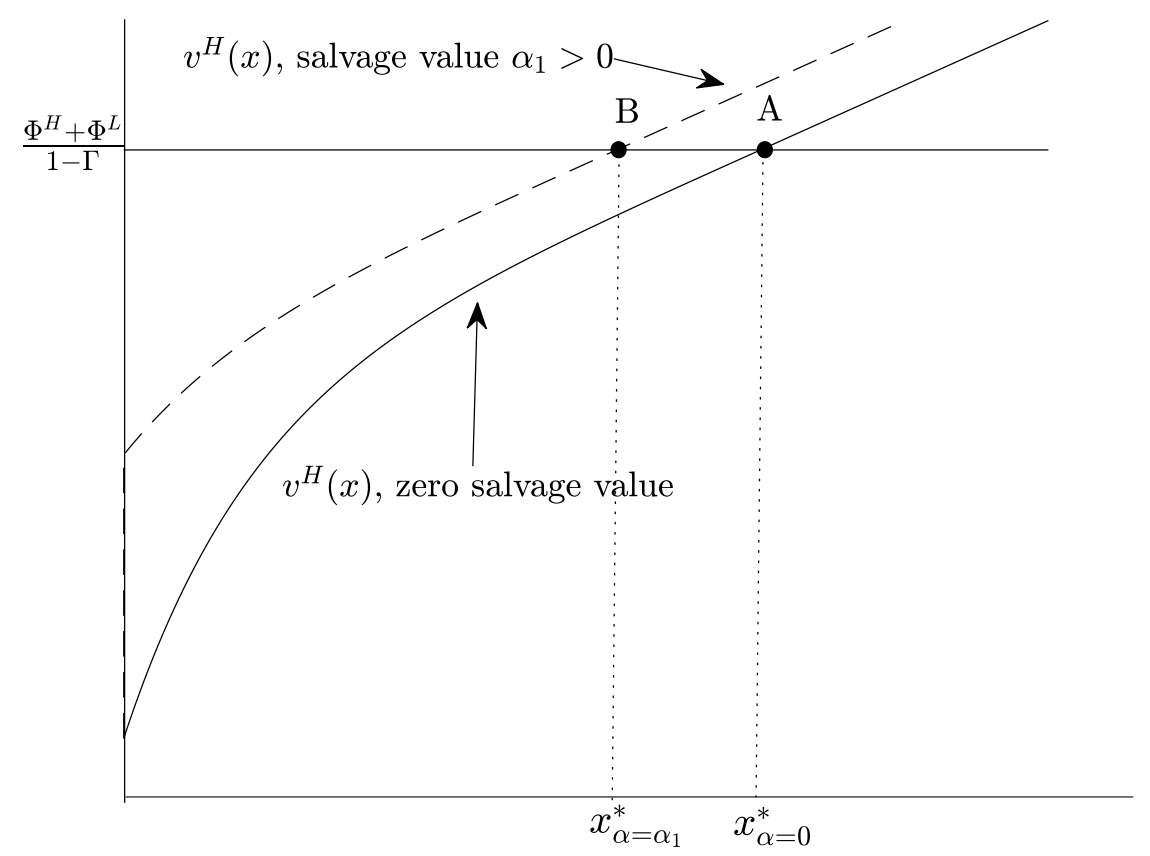

Figure 6: Impact of positive salvage value on the value function and optimal $x^{*}$.

Proposition 6 states that for any given value of $x$, an increase in the salvage value $\alpha$ increases marginal $q$, which implies that optimal investment increases also. It may seem obvious that increasing the salvage value of capital will effectively reduce the user cost of capital and thus stimulate investment, but there are actually two channels that increase investment. The first is the seemingly obvious channel, which increases the value of a unit of capital, $v^{j}(x, \alpha)$, in the numerator of $\frac{v^{j}(x, \alpha)}{v_{x}^{j}(x, \alpha)}$; the second channel is that an increase in $\alpha$ reduces the shadow price of cash on hand, $v_{x}^{j}(x, \alpha)$, which is the denominator of $\frac{v^{j}(x, \alpha)}{v_{x}^{j}(x, \alpha)}$. This reduction in the denominator further increases marginal $q$ in addition to the increase in marginal $q$ resulting from the increase in the numerator.

Figure 6 illustrates that an increase in the salvage value $\alpha$ increases the value of a unit of capital for any given value of cash on hand, $x$, and yet the value of a unit of capital evaluated at $x=x^{*}$ is invariant to $\alpha$. The upward-sloping solid curve that passes through point A shows the value of a unit of capital in Regime $H$ on the vertical axis as a function of cash on hand, $x$, on the horizontal axis. This curve is drawn for the case in which $\alpha=0$, that is, for the case with zero salvage value. At point A, with $\alpha=0, x=x_{\alpha=0}^{*}$ so the value of a unit of capital is $v^{H}\left(x^{*}\right)=\frac{\Phi^{H}+\Phi^{L}}{1-\Gamma}$ (Statement 1 of Proposition 3). At point A, the marginal 
of cash on hand, $v_{x}^{H}(x)$, equals one according the boundary condition in equation (18). At points on the solid curve that are to the left of point $\mathrm{A}$, the marginal value of cash on hand is greater than one, and at points on the solid curve that are to the right of point $\mathrm{A}$, the marginal value of cash on hand is identically equal to one.

The upward-sloping dashed line that passes through point B shows the value of a unit of capital in Regime $H$ when the salvage value $\alpha=\alpha_{1}>0$. As stated in Proposition 5 , the increase in $\alpha$ reduces $x^{*}$ from its initial value $x_{\alpha=0}^{*}$ to the lower value $x_{\alpha=\alpha_{1}}^{*}$ shown on the horizontal axis. As indicated by Statement 1 in Proposition 6, the increase in $\alpha$ from zero to a positive value increases $v^{H}(x)$ for every value of $x$. As indicated by Statement 2 in Proposition 6 , the increase in $\alpha$ from zero to a positive value reduces the marginal value of cash, $v_{x}^{H}(x)$, for every value of $x \in\left[0, x_{\alpha=0}^{*}\right)$. At point $\mathrm{B}$, where $x=x_{\alpha=\alpha_{1}}^{*}$, the value of a unit of capital is $v^{H}\left(x_{\alpha=\alpha_{1}}^{*}\right)=\frac{\Phi^{H}+\Phi^{L}}{1-\Gamma}$, just as it is point A when the salvage value is zero.

In simple economic terms, the introduction of a positive salvage value reduces the costliness of termination, so the incentive to forestall termination is reduced. Thus, the firm chooses a lower value of $x^{*}$. But the lower value of $x^{*}$ means that the firm will run out of cash at an earlier date than it would if $x^{*}$ remained equal to $x_{\alpha=0}^{*}$. Thus, the firm earns operating profits over a shorter duration of time. So, the introduction of a positive salvage value has three impacts on $v^{H}\left(x^{*}\right)$. First, the introduction of a positive salvage value directly reduces cash on hand, $x^{*} K$. Second, the reduction in $x^{*}$ shortens the firm's life and reduces the expected present value of operating profits, net of investment costs, over its lifetime. Third, the introduction of a positive salvage value means that the firm collects some positive amount $\alpha$ when it terminates, which tends to increase the value of the firm. Taken together, these three effects add up to precisely zero and there is no impact on $v^{H}\left(x^{*}\right)$.

The introduction of a positive salvage value seems to strengthen our finding that financial constraints bias upward the coefficient in a univariate regression of marginal $q$ (equivalently, the optimal investment-capital ratio) on average $q$. To see why, observe that in Figure 3, in which the salvage value $\alpha$ equals zero, all points lie below the 45 degree line along which marginal $q$ equals average $q$, with two exceptions: $q_{M}^{H}\left(x^{*}\right)=q_{A}^{H}\left(x^{*}\right)$ as shown by Point A and $q_{M}^{L}(0)=q_{A}^{L}(0)=0$ as shown by Point $\mathrm{O}$. However, with a positive salvage value, $\alpha>0, q_{M}^{L}(0)=\frac{v^{L}(0)}{v_{x}^{L}(0)}=\frac{\alpha}{v_{x}^{L}(0)}<\alpha=q_{A}^{L}(0)>0$. Therefore, if Figure 3 were redrawn for the case in which the salvage value $\alpha$ is positive, the point on the lower curve where $x=0$ in Regime $L$ would lie to the northeast of Point O, but below the 45 degree line, which tends to steepen the regression line further. 


\section{Conclusion}

Financial constraints affect the real decisions of firms, such as the capital investment decision. To examine the impact of financial constraints, we introduced a stark financial constraint that prevents an ongoing firm from using any form of external finance. Formally, this constraint is written as two constraints that prevent both cash on hand and dividends from being negative. If the firm faces negative cash flow from operations when it has zero cash on hand, then it must terminate immediately and permanently. The risk of such a liquidity shortfall provides an incentive to accumulate cash on hand, but this incentive is tempered by shareholders' preference to receive dividends sooner rather than later.

Financial constraints reduce the value of the firm, thereby reducing marginal $q$ and reducing optimal investment. Notably, the reduction in the optimal rate of investment occurs even when the financial constraints are not currently binding, but the financial constraints would not be reflected by the regression coefficient on cash flow.

The $q$-theory of investment is easily derived for a neoclassical firm that faces convex costs of adjusting its capital stock and is free of any financial constraints. A powerful implication of that theory is that marginal $q$ is a sufficient statistic for optimal investment. Marginal $q$ is not directly observable, but average $q$, which is observable, is identically equal to marginal $q$ if the Hayashi conditions hold. Thus empirical analyses appeal to the Hayashi conditions and use average $q$ as a proxy for marginal $q$ in investment regressions. When cash flow is included as a second regressor in those regressions, the coefficient on cash flow is often positive. A positive coefficient on cash flow is interpreted by many researchers as evidence of financial constraints, though other researchers interpret the positive coefficient as an artifact of classical measurement error in average $q$.

In this paper, we use the convex adjustment cost framework to examine optimal investment for a firm that satisfies the Hayashi conditions and faces the stark financial constraint described above. Though the financial constraint reduces the optimal rate of investment, it does not affect the relationship between the optimal investment-capital ratio and marginal $q$. In particular, the financial constraint does not affect marginal $q$ 's status as a sufficient statistic for investment. However, the financial constraint drives a wedge between marginal $q$ and average $q$, which has an impact on investment regressions that use average $q$ as a regressor. For univariate regressions of the investment-capital ratio on average $q$, the financial constraint biases the coefficient on average $q$ upward, rather than downward as would be the case with classical measurement error. The addition of cash flow as a second regressor 
in such investment regressions reduces the coefficient on average $q$; and, importantly, the coefficient on cash flow is positive. The positive coefficient on cash flow is evidence of a financial constraint since it is a consequence of the wedge between average $q$ and marginal $q$ induced by financial constraints of the sort we have modeled. The coefficient on cash flow comprises two components. One component reflects the financial constraint as an increasing function of the marginal valuation of cash on hand multplied by the value of the firm per unit of capital. The other component is a portion of the impact of expected future profitability on investment, which diminishes the contribution of this impact to the coefficient on average $q$. The cash flow coefficient is not simply a reflection of a liquidity shortfall that limits investment.

If the sample were to include only observations for which $D>0$, so that the financial constraint is not binding, the wedge between average $q$ and marginal $q$ would disappear. In that case, in the absence of any other source of measurement error, average $q$ would be a sufficient statistic for investment in the sample, and the coefficient on cash flow would be zero. However, one must use caution in concluding that the zero coefficient is evidence against the relevance of financial constraints. In this situation, the financial constraint is not binding, but optimal investment is, neverthess, smaller than it would be in the complete absence of financial constraints. 


\section{References}

Abel, A. (1983). Optimal investment under uncertainty. American Economic Review 73(1), $228-33$.

Abel, A. B. (2018). The effects of q and cash flow on investment in the presence of measurement error. Journal of Financial Economics 128(2), 363-377.

Abel, A. B. and J. C. Eberly (2011). How q and cash flow affect investment without frictions: An analytic explanation. The Review of Economic Studies 78(4), 1179-1200.

Abel, A. B. and S. Panageas (2019). Precautionary saving in a financially-constrained firm. Working Paper.

Alti, A. (2003). How sensitive is investment to cash flow when financing is frictionless? The Journal of Finance 58(2), 707-722.

Bolton, P., H. Chen, and N. Wang (2011). A unified theory of tobin's q, corporate investment, financing, and risk management. The Journal of Finance 66 (5), 1545-1578.

Brainard, W. C. and J. Tobin (1968). Pitfalls in financial model-building. Cowles Foundation Discussion Papers 244, Cowles Foundation for Research in Economics, Yale University.

Chirinko, R. S. (1993). Business fixed investment spending: Modeling strategies, empirical results, and policy implications. Journal of Economic Literature 31(4), 1875-1911.

Cooper, R. and J. Ejarque (2003). Financial frictions and investment: requiem in q. Review of Economic Dynamics 6(4), 710 - 728.

Erickson, T. and T. M. Whited (2000). Measurement Error and the Relationship between Investment and q. Journal of Political Economy 108(5), 1027-1057.

Fazzari, S. M., R. G. Hubbard, and B. C. Petersen (1988). Financing constraints and corporate investment. Brookings Papers on Economic Activity 1988(1), 141-195.

Gilchrist, S. and C. P. Himmelberg (1995). Evidence on the role of cash flow for investment. Journal of Monetary Economics 36(3), 541 - 572.

Gomes, J. F. (2001). Financing investment. American Economic Review 91 (5), 1263-1285. 
Hayashi, F. (1982). Tobin's marginal q and average q: A neoclassical interpretation. Econometrica 50(1), 213-224.

Hayashi, F. and T. Inoue (1991). The relation between firm growth and q with multiple capital goods: Theory and evidence from panel data on japanese firms. Econometrica 59(3), $731-753$.

Hoshi, T. and A. K. Kashyap (1990). Evidence on q and investment for japanese firms. Journal of the Japanese and International Economies 4(4), 371 - 400.

Kaplan, S. N. and L. Zingales (1997). Do investment-cash flow sensitivities provide useful measures of financing constraints? The Quarterly Journal of Economics 112(1), 169-215.

Keynes, J. M. (1936). The general theory of employment, interest, and money. Macmillan Cambridge University Press.

Mussa, M. L. (1977). External and Internal Adjustment Costs and the Theory of Aggregate and Firm Investment. Economica 44(174), 163-178.

Peters, R. H. and L. A. Taylor (2017). Intangible capital and the investment-q relation. Journal of Financial Economics 123(2), 251-272.

Poterba, J. (1988). Comment on "financing constraints and corporate investment". Brookings Papers on Economic Activity 1988(1), 200-204.

Roberts, M. R. and T. M. Whited (2013). Chapter 7 - endogeneity in empirical corporate finance1. In G. M. Constantinides, M. Harris, and R. M. Stulz (Eds.), Handbook of the Economics of Finance, Volume 2, pp. 493 - 572. Elsevier.

Summers, L. (1981). Taxation and corporate investment: a q-theory approach. Brookings Papers on Economic Activity 1981 (1), 67-127.

Tobin, J. (1969). A general equilibrium approach to monetary theory. Journal of Money, Credit and Banking 1(1), 15-29.

Whited, T. M. (1992). Debt, liquidity constraints, and corporate investment: Evidence from panel data. The Journal of Finance 47(4), 1425-1460. 


\section{Appendix}

\section{A Proofs}

Proof of Lemma 1. For any $\Delta>0, X \geq 0$, and $K>0, V^{j}(X+\Delta, K) \geq V^{j}(X, K)+\Delta$, so $\lim _{\Delta \rightarrow 0} \frac{V^{j}(X+\Delta, K)-V^{j}(X, K)}{\Delta} \geq \lim _{\Delta \rightarrow 0} \frac{\Delta}{\Delta}=1$.

Proof of Lemma 2. Since $v^{L}(x)$ is strictly increasing (Lemma 1 and equation (10)), $x^{*}>0$ if and only if $v^{L}\left(x^{*}\right)>v^{L}(0)$. Then the boundary condition in equation (20), $v^{L}(0)=\alpha$, implies that $x^{*}>0$ if and only if $v^{L}\left(x^{*}\right)>\alpha$.

Proof of Proposition 1. See paragraph immediately following Proposition 1.

Proof of Proposition 2. In Regime $H$, the HJB equation in equation (16) and the boundary condition in equation $(20), v^{L}(0)=\alpha$, together imply (Statement 1) $v^{H}(0)=$ $v_{x}^{H}(0) \Phi^{H}+\frac{\mu^{L}}{\rho+\mu^{L}} \alpha>\Phi^{H}$, where the inequality follows from $\alpha \geq 0$ and $v_{x}^{H}(0)>1$ when $x^{*}>0$. Substituting this expression for $v^{H}(0)$ into the definition $q_{M}^{H}(x) \equiv \frac{v^{H}(x)}{v_{x}^{H}(x)}-x$ and setting $x=0$ yields (Statement 2) $q_{M}^{H}(0)=\Phi^{H}+\frac{\mu^{L}}{\rho+\mu^{L}} \frac{\alpha}{v_{x}^{L}(0)} \geq \Phi^{H}$.

Now consider Regime $L$. Statement 3 is simply the boundary condition in equation $(20), v^{L}(0)=\alpha$, which together with $v_{x}^{L}(0)>1$ (from Lemma 1 and equation(10)), implies $q_{M}^{L}(0)=\frac{\alpha}{v_{x}^{L}(0)}<\alpha$.

Proof of Proposition 3. Substitute equation (29) into equation (28) to obtain $v^{H}\left(x^{*}\right)=$ $\Phi^{H}+\frac{\mu^{L}}{\rho+\mu^{L}}\left[\frac{\rho+\mu^{L}}{\mu^{L}} \Phi^{L}+\frac{\mu^{H}}{\rho+\mu^{H}} v^{H}\left(x^{*}\right)\right]=\Phi^{H}+\Phi^{L}+\Gamma v^{H}\left(x^{*}\right)$, where $\Gamma \equiv \frac{\mu^{H}}{\rho+\mu^{H}} \frac{\mu^{L}}{\rho+\mu^{L}}$. Therefore, (Statement 1) $v^{H}\left(x^{*}\right)=\frac{1}{1-\Gamma}\left[\Phi^{H}+\Phi^{L}\right]<\frac{1}{1-\Gamma}\left[\Phi^{H}+\frac{\mu^{L}}{\rho+\mu^{L}} \Phi^{L}\right]=\widetilde{v}^{H}$, where the inequality follows from $\Gamma<1, \frac{\mu^{L}}{\rho+\mu^{L}}<1$, and $\Phi^{L}<0$. Use the boundary condition $v_{x}^{H}\left(x^{*}\right)=1$ from equation (18) and the definition $q_{M}^{H}(x) \equiv \frac{v^{H}(x)}{v_{x}^{H}(x)}-x$ from equation (14) to obtain (Statement 2) $q_{M}^{H}(x)=v^{H}\left(x^{*}\right)-x^{*}=\frac{\Phi^{H}+\Phi^{L}}{1-\Gamma}-x^{*}<\widetilde{v}^{H}$, where the second equality and the inequality follow from Statement 1. Substitute equation (28) into equation (29) to obtain $v^{L}\left(x^{*}\right)=\frac{\rho+\mu^{L}}{\mu^{L}} \Phi^{L}+$ $\left[\frac{\mu^{H}}{\rho+\mu^{H}} \Phi^{H}+\frac{\mu^{H}}{\rho+\mu^{H}} \frac{\mu^{L}}{\rho+\mu^{L}} v^{L}\left(x^{*}\right)\right]=\frac{\rho+\mu^{L}}{\mu^{L}}\left[\Phi^{L}+\Gamma \Phi^{H}\right]+\Gamma v^{L}\left(x^{*}\right) . \quad$ Therefore, (Statement 3) $v^{L}\left(x^{*}\right)=\frac{1}{1-\Gamma} \frac{\rho+\mu^{L}}{\mu^{L}}\left[\Phi^{L}+\Gamma \Phi^{H}\right]<\frac{1}{1-\Gamma}\left[\Phi^{L}+\frac{\mu^{H}}{\rho+\mu^{H}} \Phi^{H}\right]=\widetilde{v}^{L}$, where the inequality follows from $\frac{\rho+\mu^{L}}{\mu^{L}}>1, \Phi^{L}<0$, and $\frac{\rho+\mu^{L}}{\mu^{L}} \Gamma=\frac{\mu^{H}}{\rho+\mu^{H}}$. Use $v_{x}^{L}\left(x^{*}\right)=\frac{\rho+\mu^{L}}{\mu^{L}}$ from equation (27) and $q_{M}^{L}(x) \equiv \frac{v^{L}(x)}{v_{x}^{L}(x)}-x$ to obtain (Statement 4) $q_{M}^{L}\left(x^{*}\right)=\frac{v^{L}\left(x^{*}\right)}{v_{x}^{L}\left(x^{*}\right)}-x^{*}=\frac{\Phi^{L}+\Gamma \Phi^{H}}{1-\Gamma}-x^{*}<\widetilde{v}^{L}$, where the second equality and the inequality follow from Statement 3 .

Proof of Corollary 1. Assume that $x^{*}>0$ and consider $0 \leq x \leq x^{*}$. Then $q_{M}^{j}(x) \leq$ $q_{M}^{j}\left(x^{*}\right)=\frac{v^{j}\left(x^{*}\right)}{v_{x}^{j}\left(x^{*}\right)}-x^{*}<v^{j}\left(x^{*}\right)<\widetilde{v}^{j}$ where the first inequality follows from the fact that 
$q_{M}^{j}(x)$ is increasing in $x$ for $0 \leq x \leq x^{*}$, the equality is simply the definition of $q_{M}^{j}(x)$ evaluated at $x=x^{*}$, the second inequality follows from $v_{x}^{j}\left(x^{*}\right) \geq 1$ and $x^{*}>0$ and the final inequality follows from Statements 1 and 3 of Proposition 3.

Proof of Proposition 4. Lemma 2 and Statement 3 of Proposition 3 imply $x^{*}>0 \Longleftrightarrow$ $\frac{\rho+\mu^{L}}{\mu^{L}} \frac{\Phi^{L}+\Gamma \Phi^{H}}{1-\Gamma}>\alpha \Longleftrightarrow \frac{\Phi^{L}+\Gamma \Phi^{H}}{1-\Gamma}>\frac{\mu^{L}}{\rho+\mu^{L}} \alpha$. Observe that $\frac{\Phi^{L}+\Gamma \Phi^{H}}{1-\Gamma}=\frac{\Phi^{L}+\Phi^{H}}{1-\Gamma}-\Phi^{H}=v^{H}\left(x^{*}\right)-$ $\Phi^{H}$, where the second equality follows from Statement 1 of Proposition 3. Therefore, $x^{*}>0 \Longleftrightarrow v^{H}\left(x^{*}\right)-\Phi^{H}>\frac{\mu^{L}}{\rho+\mu^{L}} \alpha$, or equivalently, $v^{H}\left(x^{*}\right)>\Phi^{H}+\frac{\mu^{L}}{\rho+\mu^{L}} \alpha$.

\section{Proof of Proposition 5.}

Since $u \equiv x-x^{*}, v^{L}(0)=g^{L}\left(-x^{*}\right)$. Therefore, the boundary condition $v^{L}(0)=\alpha$ (equation 20) can be written as $g\left(-x^{*}\right)=\alpha$, which can be implicitly differentiated to obtain $\frac{d x^{*}}{d \alpha}=-\frac{1}{g^{\prime}\left(-x^{*}\right)}=-\frac{1}{v_{x}^{L}(0)}<0$.

Proof of Proposition 6. Differentiate equation (43) with respect to $x$ obtain $v_{x}^{j}(x, \alpha)=$ $g^{j \prime}\left(x-x^{*}(\alpha)\right)>0$, where the inequality follows from Lemma 1. Differentiate equation (43) with respect to $\alpha$ to obtain $v_{\alpha}^{j}(x, \alpha)=-g^{j \prime}\left(x-x^{*}(\alpha)\right) x^{* \prime}(\alpha)>0$, where the inequality follows from Proposition 5 and $v_{x}^{j}(x, \alpha)=g^{j \prime}\left(x-x^{*}(\alpha)\right)>0$. Therefore, an increase in $\alpha$ increases the value of a unit capital, $v^{j}(x, \alpha)$, at any given level of cash on hand in either regime.

To determine the impact of $\alpha$ on the marginal valuation of cash in Regime $j, v_{x}^{j}$, first differentiate $v_{x}^{j}(x, \alpha)=g^{j \prime}\left(x-x^{*}(\alpha)\right)$ with respect to $x$ to obtain $v_{x x}^{j}(x, \alpha)=g^{j \prime \prime}\left(x-x^{*}(\alpha)\right) \leq$ 0 , for $0 \leq x \leq x^{*}$, where the inequality follows from the concavity of $v^{j}(x)$ for $0 \leq x \leq x^{*}$ and is strict for $0 \leq x<x^{*}$. Now differentiate $v_{x}^{j}(x, \alpha)=g^{j \prime}\left(x-x^{*}(\alpha)\right)$ with respect to $\alpha$ to obtain $v_{x \alpha}^{j}(x, \alpha)=-g^{j \prime \prime}\left(x-x^{*}(\alpha)\right) x^{* \prime}(\alpha) \leq 0$, for $0 \leq x \leq x^{*}$, where the inequality follows from Proposition 5 and $v_{x x}^{j}(x, \alpha)=g^{j \prime \prime}\left(x-x^{*}(\alpha)\right) \leq 0$. Therefore, an increase in $\alpha$ reduces the marginal valuation of cash, $v_{x}^{j}(x, \alpha)$, at any given value of $x$.

Differentiate $q_{M}^{j}(x, \alpha) \equiv \frac{v^{j}(x, \alpha)}{v_{x}^{j}(x, \alpha)}-x$ with respect to $\alpha$ to obtain $\frac{\partial q_{M}^{j}(x, \alpha)}{\partial \alpha}=\frac{v_{a}^{j}(x, \alpha)}{v_{x}^{j}(x, \alpha)}-$ $\frac{v^{j}(x, \alpha)}{\left[v_{x}^{j}(x, \alpha)\right]^{2}} v_{x \alpha}^{j}(x, \alpha)>0$ for $0 \leq x \leq x^{*}$. 


\section{B Solution of the ODEs in Equations (16) and (17) and boundary conditions conditions in equations (18), (19), and (20)}

This appendix first presents, and then verifies, the solutions to the ODEs in equations (16) and (17) and boundary conditions conditions in equations (18), (19), and (20). The solutions for the value of the firm in Regime $H$ and Regime $L$ are in equations (B.1) and (B.2), respectively. The solution for $x^{*}$ is the root of $h(u)=0$ where $h(u)$ is defined in equation (B.6), provided that $\frac{\Phi^{L}+\Gamma \Phi^{H}}{1-\Gamma}>\frac{\mu^{L}}{\rho+\mu^{L}} \alpha$ so that (from Proposition 4) $x^{*}>0$.

$$
v^{H}(x)=c_{1} e^{\omega_{1}\left(x-x^{*}\right)}+c_{2} e^{\omega_{2}\left(x-x^{*}\right)}
$$

and

$$
v^{L}(x)=c_{1} \frac{\rho+\mu^{L}}{\mu^{L}}\left(1-\omega_{1} \Phi^{H}\right) e^{\omega_{1}\left(x-x^{*}\right)}+c_{2} \frac{\rho+\mu^{L}}{\mu^{L}}\left(1-\omega_{2} \Phi^{H}\right) e^{\omega_{2}\left(x-x^{*}\right)}
$$

where $\omega_{1}<0<\omega_{2}<\frac{1}{\Phi^{H}}$ are the roots of the characteristic equation ${ }^{25}$

$$
q(\omega) \equiv \omega^{2}-\left(\frac{1}{\Phi^{H}}+\frac{1}{\Phi^{L}}\right) \omega+\frac{1}{\Phi^{H}} \frac{1}{\Phi^{L}}(1-\Gamma)=0, \quad i=1,2
$$

and

$$
c_{1}=\frac{1}{\omega_{2}-\omega_{1}} \frac{\omega_{2}}{\omega_{1}}<0
$$

and

$$
c_{2}=\frac{1}{\omega_{1}-\omega_{2}} \frac{\omega_{1}}{\omega_{2}}>0 .
$$

If $\frac{\Phi^{L}+\Gamma \Phi^{H}}{1-\Gamma}>\frac{\mu^{L}}{\rho+\mu^{L}} \alpha$, then $x^{*}$ is the unique positive root of $h(u)=0$, where ${ }^{26}$

$$
h(u) \equiv c_{1}\left(1-\omega_{1} \Phi^{H}\right) e^{-\omega_{1} u}+c_{2}\left(1-\omega_{2} \Phi^{H}\right) e^{-\omega_{2} u}-\frac{\mu^{L}}{\rho+\mu^{L}} \alpha .
$$

\footnotetext{
${ }^{25}$ Since $q^{\prime \prime}(\omega)>0$ and $q(0)=\frac{1}{\Phi^{H}} \frac{1}{\Phi^{L}}(1-\Gamma)<0, \omega_{1}<0<\omega_{2}$. In addition, since $q\left(\frac{1}{\Phi^{H}}\right)=-\frac{1}{\Phi^{H}} \frac{1}{\Phi^{L}} \Gamma>0$, $\omega_{2}<\frac{1}{\Phi^{H}}$.

${ }^{26}$ Since $\omega_{1}+\omega_{2}=\frac{1}{\Phi^{H}}+\frac{1}{\Phi^{L}}$ and $\omega_{1} \omega_{2}=\frac{1}{\Phi^{H}} \frac{1}{\Phi^{L}}(1-\Gamma)$, it follows that $c_{1}+c_{2}=\frac{1}{\omega_{2}-\omega_{1}}\left(\frac{\omega_{2}}{\omega_{1}}-\frac{\omega_{1}}{\omega_{2}}\right)=$ $\frac{\omega_{2}^{2}-\omega_{1}^{2}}{\omega_{2}-\omega_{1}} \frac{1}{\omega_{1} \omega_{2}}=\frac{\omega_{1}+\omega_{2}}{\omega_{1} \omega_{2}}=\frac{\frac{1}{\Phi^{H}}+\frac{1}{\Phi^{L}}}{\frac{1}{\Phi^{H}} \frac{1}{\Phi^{L}}(1-\Gamma)}=\frac{\Phi^{H}+\Phi^{L}}{1-\Gamma}$. Use this expression for $c_{1}+c_{2}$ and the fact that $c_{1} \omega_{1}+c_{2} \omega_{2}=$ 1 to obtain $h(0)=c_{1}\left(1-\omega_{1} \Phi^{H}\right)+c_{2}\left(1-\omega_{2} \Phi^{H}\right)-\frac{\mu^{L}}{\rho+\mu^{L}} \alpha=c_{1}+c_{2}-\left(c_{1} \omega_{1}+c_{2} \omega_{2}\right) \Phi^{H}-\frac{\mu^{L}}{\rho+\mu^{L}} \alpha=$ $\frac{\Phi^{H}+\Phi^{L}}{1-\Gamma}-\Phi^{H}-\frac{\mu^{L}}{\rho+\mu^{L}} \alpha=\frac{\Phi^{L}+\Gamma \Phi^{H}}{1-\Gamma}-\frac{\mu^{L}}{\rho+\mu^{L}} \alpha$ will be positive if and only if $\frac{\Phi^{L}+\Gamma \Phi^{H}}{1-\Gamma}-\frac{\mu^{L}}{\rho+\mu^{L}} \alpha>0$ which is the case if and only if $x^{*}>0$. Differentiate the expression for $h(u)$ in equation (B.6) to obtain $h^{\prime}(u)=$ $-c_{1} \omega_{1}\left(1-\omega_{1} \Phi^{H}\right) e^{-\omega_{1} u}-c_{2} \omega_{2}\left(1-\omega_{2} \Phi^{H}\right) e^{-\omega_{2} u}<0$. Since we are assuming that $\frac{\Phi^{L}+\Gamma \Phi^{H}}{1-\Gamma}-\frac{\mu^{L}}{\rho+\mu^{L}} \alpha>0$, we have $h(0)>0$ and $h^{\prime}(u)<0$, so $h(u)=0$ has a unique positive root.
} 
In the special case in which the salvage value, $\alpha$, equals zero, there is a closed-form expression for $x^{*}$.

$$
x^{*}=\max \left[\frac{1}{\omega_{2}-\omega_{1}} \ln (1+\Omega), 0\right], \quad \text { if } \alpha=0
$$

where

$$
\Omega \equiv \frac{\Phi^{L}+\Gamma \Phi^{H}}{1-\Gamma-\left(\Phi^{L}+\Gamma \Phi^{H}\right) \omega_{2}}\left(\omega_{2}-\omega_{1}\right) .
$$

To verify the solutions in equations (B.1) and (B.2), first differentiate each of these equations with respect to $x$ to obtain

$$
v_{x}^{H}(x)=\omega_{1} c_{1} e^{\omega_{1}\left(x-x^{*}\right)}+\omega_{2} c_{2} e^{\omega_{2}\left(x-x^{*}\right)}
$$

and

$$
v_{x}^{L}(x)=\omega_{1} c_{1} \frac{\rho+\mu^{L}}{\mu^{L}}\left(1-\omega_{1} \Phi^{H}\right) e^{\omega_{1}\left(x-x^{*}\right)}+\omega_{2} c_{2} \frac{\rho+\mu^{L}}{\mu^{L}}\left(1-\omega_{2} \Phi^{H}\right) e^{\omega_{2}\left(x-x^{*}\right)} .
$$

Use equations (B.2) and (B.9) to write the right hand side of equation (16) as

$$
\begin{aligned}
v_{x}^{H}(x) \Phi^{H}+\frac{\mu^{L}}{\rho+\mu^{L}} v^{L}(x)= & {\left[\omega_{1} c_{1} e^{\omega_{1}\left(x-x^{*}\right)}+\omega_{2} c_{2} e^{\omega_{2}\left(x-x^{*}\right)}\right] \Phi^{H} } \\
& +\frac{\mu^{L}}{\rho+\mu^{L}}\left[\begin{array}{c}
c_{1} \frac{\rho+\mu^{L}}{\mu^{L}}\left(1-\omega_{1} \Phi^{H}\right) e^{\omega_{1}\left(x-x^{*}\right)} \\
+c_{2} \frac{\rho+\mu^{L}}{\mu^{L}}\left(1-\omega_{2} \Phi^{H}\right) e^{\omega_{2}\left(x-x^{*}\right)}
\end{array}\right]
\end{aligned}
$$

which simplifies to

$$
v_{x}^{H}(x) \Phi^{H}+\frac{\mu^{L}}{\rho+\mu^{L}} v^{L}(x)=c_{1} e^{\omega_{1}\left(x-x^{*}\right)}+c_{2} e^{\omega_{2}\left(x-x^{*}\right)} .
$$

Note that the right hand side equation (B.11) equals the expression for $v^{H}(x)$ in equation (B.1).

Use equations (B.1) and (B.10) to write the right hand side of equation (17) as

$$
\begin{aligned}
v_{x}^{L}(x) \Phi^{L}+\frac{\mu^{H}}{\rho+\mu^{H}} v^{H}(x)= & {\left[\begin{array}{c}
\omega_{1} c_{1} \frac{\rho+\mu^{L}}{\mu^{L}}\left(1-\omega_{1} \Phi^{H}\right) e^{\omega_{1}\left(x-x^{*}\right)} \\
+\omega_{2} c_{2} \frac{\rho+\mu^{L}}{\mu^{L}}\left(1-\omega_{2} \Phi^{H}\right) e^{\omega_{2}\left(x-x^{*}\right)}
\end{array}\right] \Phi^{L} } \\
& +\frac{\mu^{H}}{\rho+\mu^{H}}\left[c_{1} e^{\omega_{1}\left(x-x^{*}\right)}+c_{2} e^{\omega_{2}\left(x-x^{*}\right)}\right]
\end{aligned}
$$


which, using $\Gamma \equiv \frac{\mu^{L}}{\rho+\mu^{L}} \frac{\mu^{H}}{\rho+\mu^{H}}$, simplifies to

$$
v_{x}^{L}(x) \Phi^{L}+\frac{\mu^{H}}{\rho+\mu^{H}} v^{H}(x)=\frac{\rho+\mu^{L}}{\mu^{L}}\left[\begin{array}{c}
{\left[\omega_{1} \Phi^{L}\left(1-\omega_{1} \Phi^{H}\right)+\Gamma\right] c_{1} e^{\omega_{1}\left(x-x^{*}\right)}} \\
+\left[\omega_{2} \Phi^{L}\left(1-\omega_{2} \Phi^{H}\right)+\Gamma\right] c_{2} e^{\omega_{2}\left(x-x^{*}\right)}
\end{array}\right] .
$$

Evaluating the characteristic function in equation (B.3) at the roots $\omega_{i}, i=1,2$, yields $\Phi^{H} \Phi^{L} \omega_{i}^{2}-\left(\Phi^{L}+\Phi^{H}\right) \omega_{i}+1-\Gamma=0$, which implies $\Gamma+\Phi^{L} \omega_{i}-\Phi^{H} \Phi^{L} \omega_{i}^{2}=1-\Phi^{H} \omega_{i}$, equivalently,

$$
\omega_{i} \Phi^{L}\left(1-\omega_{i} \Phi^{H}\right)+\Gamma=1-\Phi^{H} \omega_{i}, \quad i=1,2 .
$$

Use equation (B.13) to rewrite equation (B.12) as

$$
v_{x}^{L}(x) \Phi^{L}+\frac{\mu^{H}}{\rho+\mu^{H}} v^{H}(x)=\frac{\rho+\mu^{L}}{\mu^{L}}\left[\begin{array}{c}
\left(1-\Phi^{H} \omega_{1}\right) c_{1} e^{\omega_{1}\left(x-x^{*}\right)} \\
+\left[1-\Phi^{H} \omega_{2}\right] c_{2} e^{\omega_{2}\left(x-x^{*}\right)}
\end{array}\right] .
$$

Note that the right hand side equation (B.14) equals the expression for $v^{L}(x)$ in equation (B.2). Thus, we have verified that the expressions for $v^{H}(x)$ and $v^{L}(x)$ in equations (B.1) and (B.2) satisfy the ODEs in equations (16) and (17), respectively.

Next we verify the boundary conditions. Differentiate $v^{H}(x)$ in equation (B.1) twice with respect to $x$, evaluate $v_{x}^{H}(x)$ and $v_{x x}^{H}(x)$ at $x=x^{*}$, and use the definitions of $c_{1}$ and $c_{2}$ in equations (B.4) and (B.5) to obtain

$$
v_{x}^{H}\left(x^{*}\right)=c_{1} \omega_{1}+c_{2} \omega_{2}=1
$$

and

$$
v_{x x}^{H}\left(x^{*}\right)=c_{1} \omega_{1}^{2}+c_{2} \omega_{2}^{2}=0,
$$

which are the boundary conditions in equations (18) and (19), respectively.

Evaluate $v^{L}(x)$ in equation (B.2) at $x=0$, to obtain

$$
v^{L}(0)=c_{1} \frac{\rho+\mu^{L}}{\mu^{L}}\left(1-\omega_{1} \Phi^{H}\right) e^{-\omega_{1} x^{*}}+c_{2} \frac{\rho+\mu^{L}}{\mu^{L}}\left(1-\omega_{2} \Phi^{H}\right) e^{-\omega_{2} x^{*}},
$$

which can be rewritten as

$$
v^{L}(0)=\frac{\rho+\mu^{L}}{\mu^{L}}\left[h\left(x^{*}\right)+\frac{\mu^{L}}{\rho+\mu^{L}} \alpha\right]=\alpha,
$$

where the second equality follows from $h\left(x^{*}\right)=0$. Equation (B.18) is equivalent to the boundary condition in equation (20). 
For the special case in which $\alpha=0$, rearrange equation (B.17) using $\frac{c_{1}}{c_{2}}=-\frac{\omega_{2}^{2}}{\omega_{1}^{2}}$, which is implied by equations (B.4) and (B.5), to obtain

$$
e^{\left(\omega_{2}-\omega_{1}\right) x^{*}}=Z \equiv \frac{\omega_{1}^{2}}{\omega_{2}^{2}} \frac{1-\omega_{2} \Phi^{H}}{1-\omega_{1} \Phi^{H}}>0, \quad \text { if } \alpha=0 .
$$

The fact that $Z>0$ in equation (B.19) follows from $\omega_{1}<0<\omega_{2}<\frac{1}{\Phi^{H}}$. Take the $\ln$ of both sides of equation (B.19) to obtain

$$
x^{*}=\frac{1}{\omega_{2}-\omega_{1}} \ln Z, \text { if } Z \geq 1 \text { and } \alpha=0 .
$$

Evaluate the characteristic polynominal at the roots $\omega_{i}$ and $\omega_{j}, i \neq j$, to obtain ${ }^{27}$

$$
\omega_{1}^{2}\left(1-\omega_{2} \Phi^{H}\right)=\frac{-1}{\Phi^{H} \Phi^{L}}\left[1-\Gamma-\left(\Phi^{L}+\Gamma \Phi^{H}\right) \omega_{1}\right]
$$

and

$$
\omega_{2}^{2}\left(1-\omega_{1} \Phi^{H}\right)=\frac{-1}{\Phi^{H} \Phi^{L}}\left[1-\Gamma-\left(\Phi^{L}+\Gamma \Phi^{H}\right) \omega_{2}\right] .
$$

Use equations (B.21) and (B.22) to rewrite the definition of $Z$ in equation (B.19) as

$$
Z \equiv \frac{1-\Gamma-\left(\Phi^{L}+\Gamma \Phi^{H}\right) \omega_{1}}{1-\Gamma-\left(\Phi^{L}+\Gamma \Phi^{H}\right) \omega_{2}} .
$$

Add and subtract $\left(\Phi^{L}+\Gamma \Phi^{H}\right) \omega_{2}$ in the numerator of $Z$ in equation (B.23) and rearrange to obtain

$$
Z=1+\frac{\left(\Phi^{L}+\Gamma \Phi^{H}\right)\left(\omega_{2}-\omega_{1}\right)}{1-\Gamma-\left(\Phi^{L}+\Gamma \Phi^{H}\right) \omega_{2}}=1+\Omega
$$

where the second equality uses the definition of $\Omega$ in equation (B.8). Observe that if $\alpha=0$, then $x^{*}>0$ if and only if $Z>1$, which is the case if and only if $\Phi^{L}+\Gamma \Phi^{H}>0 .{ }^{28}$

Repeated differentiation of equations (B.1) and (B.2) with respect to $x$ leads to the following expressions for $v_{(j)}^{H}(x)$ and $v_{(j)}^{L}(x)$, which are the $j$-th order derivatives of $v^{H}(x)$ and $v^{L}(x)$, respectively, with respect to $x$.

$$
v_{(j)}^{H}(x)=c_{1} \omega_{1}^{j} e^{\omega_{1}\left(x-x^{*}\right)}+c_{2} \omega_{2}^{j} e^{\omega_{2}\left(x-x^{*}\right)}
$$

\footnotetext{
${ }^{27} \omega_{i}^{2}\left(1-\omega_{j} \Phi^{H}\right)=\omega_{i}^{2}-\omega_{i}^{2} \omega_{j} \Phi^{H}$. Equation (B.3) implies $\omega_{i}^{2}=\left(\frac{1}{\Phi^{H}}+\frac{1}{\Phi^{L}}\right) \omega_{i}-\frac{1}{\Phi^{H}} \frac{1}{\Phi^{L}}(1-\Gamma)$ and $\omega_{i} \omega_{j}$ $=\frac{1}{\Phi^{H}} \frac{1}{\Phi^{L}}(1-\Gamma), \quad i \neq j$, so $\omega_{i}^{2}\left(1-\omega_{j} \Phi^{H}\right)=\left[\left(\frac{1}{\Phi^{H}}+\frac{1}{\Phi^{L}}\right) \omega_{i}-\frac{1}{\Phi^{H}} \frac{1}{\Phi^{L}}(1-\Gamma)\right]-\omega_{i} \Phi^{H} \frac{1}{\Phi^{H}} \frac{1}{\Phi^{L}}(1-\Gamma)$ $=\frac{1}{\Phi^{H}} \frac{1}{\Phi^{L}}\left[\left(\Phi^{L}+\Phi^{H}\right) \omega_{i}-(1-\Gamma)-\omega_{i} \Phi^{H}(1-\Gamma)\right]=\frac{1}{\Phi^{H}} \frac{1}{\Phi^{L}}\left[\left(\Phi^{L}+\Gamma \Phi^{H}\right) \omega_{i}-(1-\Gamma)\right]=$ $-\frac{1}{\Phi^{H}} \frac{1}{\Phi^{L}}\left[1-\Gamma-\left(\Phi^{L}+\Gamma \Phi^{H}\right) \omega_{i}\right]$.

${ }^{28}$ This statement requires $1-\Gamma-\left(\Phi^{L}+\Gamma \Phi^{H}\right) \omega_{2}>0$. Since $\omega_{1}<0<\omega_{2}$ and $\Phi^{H}>0$, the left hand side of equation (B.22) is positive. In addition, since $\Phi^{L}<0$, the right hand side of equation (B.22) is positive if and only if $1-\Gamma-\left(\Phi^{L}+\Gamma \Phi^{H}\right) \omega_{2}>0$.
} 


$$
v_{(j)}^{L}(x)=\frac{\rho+\mu^{L}}{\mu^{L}}\left(1-\omega_{1} \Phi^{H}\right) c_{1} \omega_{1}^{j} e^{\omega_{1}\left(x-x^{*}\right)}+\frac{\rho+\mu^{L}}{\mu^{L}}\left(1-\omega_{2} \Phi^{H}\right) c_{2} \omega_{2}^{j} e^{\omega_{2}\left(x-x^{*}\right)} .
$$

Since $c_{1}<0, c_{2}>0$ (equations B.4 and B.5) and $\omega_{1}<0<\omega_{2}<\frac{1}{\Phi^{H}}$, it follows that $v_{(j)}^{H}(x)>0$ and $v_{(j)}^{L}(x)>0$ for $j=1,3,5, \ldots$ In particular, the third derivatives of the value functions $v^{H}(x)$ and $v^{L}(x)$ both positive.

Since $v_{x x x}^{H}(x)>0$, it follows that $v_{x x}^{H}(x)<v_{x x}^{H}\left(x^{*}\right)=0$ for $0 \leq x<x^{*}$. Therefore, $v^{H}(x)$ is strictly concave for $0 \leq x<x^{*}$. Use equations (B.25) and (B.26) to obtain

$$
v_{(j)}^{L}(x)=\frac{\rho+\mu^{L}}{\mu^{L}}\left[v_{(j)}^{H}(x)-\omega_{1} \Phi^{H} c_{1} \omega_{1}^{j} e^{\omega_{1}\left(x-x^{*}\right)}-\omega_{2} \Phi^{H} c_{2} \omega_{2}^{j} e^{\omega_{2}\left(x-x^{*}\right)}\right] .
$$

Evaluate equation (B.27) for $j=2$ at $x=x^{*}$, and use $v_{x x}^{H}\left(x^{*}\right)=0$ to obtain

$$
v_{x x}^{L}\left(x^{*}\right)=-\Phi^{H} \frac{\rho+\mu^{L}}{\mu^{L}}\left(\omega_{1}^{3} c_{1}+\omega_{2}^{3} c_{2}\right)<0 .
$$

Since $v_{x x x}^{L}(x)>0$, we have $v_{x x}^{L}(x)<v_{x x}^{L}\left(x^{*}\right)<0$ for $0 \leq x \leq x^{*}$. Therefore, $v^{L}(x)$ is strictly concave for $0 \leq x \leq x^{*}$.

\section{The Cumulative Distribution Function of $x$}

The endogenous level of cash on hand, $x_{t}$, does not have a stationary distribution because the firm eventually will encounter a Regime $L$ that lasts long enough to deplete cash on hand and force termination of the firm. Instead of stationary distribution, we focus on the distribution of $x_{t}$ over the time interval extending from the firm's birth during a Regime $H$ at a time normalized to be 0 until the firm's death at time $\tau \equiv \min \left\{t>0: x_{t}=0\right\}$. To derive the distribution function $F(x, j), j \in\{H, L\}$, we use indicator functions to indicate at each $t \in[0, \tau]$ which regime prevails and whether $x_{t}<\Delta$ for $0 \leq \Delta \leq x^{*}$.

Let $g^{H}(x)$ denote an arbitrary indicator function and define

$$
y^{H}\left(x_{t}\right) \equiv E_{t}\left\{\int_{t}^{\tau} g^{H}(z) d z\right\}
$$

which is the measure of time that the indicator function $g^{H}(z)=1$ during the interval of time from $t$ to $\tau$ given that Regime $H$ prevails at time $t$ when cash on hand is $x_{t}$. Similarly, define

$$
y^{L}\left(x_{t}\right) \equiv E_{t}\left\{\int_{t}^{\tau} g^{L}(z) d z\right\} .
$$


Setting the expected change of the left hand side of equation (C.1) equal to the expected change of the right hand side of equation (C.1) yields

$$
y_{x}^{H}\left(x_{t}\right) \phi^{H}+\mu^{L}\left(y^{L}\left(x_{t}\right)-y^{H}\left(x_{t}\right)\right)=-g^{H}\left(x_{t}\right) .
$$

Similarly,

$$
y_{x}^{L}\left(x_{t}\right) \phi^{L}+\mu^{H}\left(y^{H}\left(x_{t}\right)-y^{L}\left(x_{t}\right)\right)=-g^{L}\left(x_{t}\right) .
$$

Rearranging equations (C.3) and (C.4), and stacking them together yields

$$
\left(\begin{array}{c}
y_{x}^{H}(x) \\
y_{x}^{L}(x)
\end{array}\right)=\left(\begin{array}{cc}
\frac{\mu^{L}}{\phi^{H}} & -\frac{\mu^{L}}{\phi^{H}} \\
-\frac{\mu^{H}}{\phi^{L}} & \frac{\mu^{H}}{\phi^{L}}
\end{array}\right)\left(\begin{array}{c}
y^{H}(x) \\
y^{L}(x)
\end{array}\right)+\left(\begin{array}{c}
-\frac{1}{\phi^{H}} g^{H}(x) \\
-\frac{1}{\phi^{L}} g^{L}(x)
\end{array}\right) .
$$

Associated with the system of ODEs are the boundary conditions

$$
y_{x}^{H}\left(x^{*}\right)=0
$$

and

$$
y^{L}(0)=0
$$

The boundary condition in equation (C.6), $y_{x}^{H}\left(x^{*}\right)=0$, reflects the fact that an optimizing firm will not accumulate cash greater than $x^{*}$. If for some reason, it were to find itself with $x_{t}>x^{*}$, it would pay an immediate dividend of $x_{t}-x^{*}$. Thus, having $x_{t}>x^{*}$ would not extend the firm's liftetime, and would leave $\tau$ unchanged. Therefore, $y_{x}^{H}\left(x^{*}\right)=0$. The boundary condition in equation (C.7), $y^{L}(0)=0$, simply reflects the fact that if the firm has zero cash on hand while Regime $L$ prevails, it will terminate immediately.

Define $m^{H} \equiv \frac{\mu^{L}}{\phi^{H}}>0$ and $m^{L} \equiv \frac{\mu^{H}}{\phi^{L}}<0$. Assume that the unconditional mean of $\phi$ is positive, which implies that $\mu^{H} \phi^{H}+\mu^{L} \phi^{L}>0$. Therefore, since $\phi^{H} \phi^{L}<0$ and $m^{H}+m^{L}=\frac{1}{\phi^{H} \phi^{L}}\left(\mu^{L} \phi^{L}+\mu^{H} \phi^{H}\right)$, it follows that

$$
m^{H}+m^{L}<0 \text {. }
$$

Rewrite equation (C.5) as

$$
Y_{x}(x)=M Y(x)+G(x)
$$

where

$$
Y(x) \equiv\left(\begin{array}{c}
y^{H}(x) \\
y^{L}(x)
\end{array}\right)
$$




$$
M \equiv\left(\begin{array}{cc}
m^{H} & -m^{H} \\
-m^{L} & m^{L}
\end{array}\right)
$$

and

$$
G(x) \equiv\left(\begin{array}{c}
-\frac{1}{\phi^{H}} g^{H}(x) \\
-\frac{1}{\phi^{L}} g^{L}(x)
\end{array}\right)
$$

Let $\lambda_{1} \leq \lambda_{2}$ be the eigenvalues of $M$. Since $\lambda_{1}+\lambda_{2}=\operatorname{tr} M=m^{H}+m^{L}$ and $\lambda_{1} \lambda_{2}=$ $\operatorname{det} M=0$, we have

$$
\lambda_{1}=m^{H}+m^{L}<0
$$

and

$$
\lambda_{2}=0
$$

$\left(\begin{array}{c}m^{H} \\ -m^{L}\end{array}\right)$ is an eigenvector of $M$ with eigenvalue $\lambda_{1}=m^{H}+m^{L}$ and $\left(\begin{array}{c}1 \\ 1\end{array}\right)$ is an eigenvector of $M$ with eigenvalue $\lambda_{2}=0$.

Diagonalize $M$ using

$$
\Lambda \equiv\left(\begin{array}{cc}
m^{H}+m^{L} & 0 \\
0 & 0
\end{array}\right)
$$

and

$$
P \equiv\left(\begin{array}{cc}
m^{H} & 1 \\
-m^{L} & 1
\end{array}\right)
$$

so

$$
P^{-1}=\frac{1}{m^{H}+m^{L}}\left(\begin{array}{cc}
1 & -1 \\
m^{L} & m^{H}
\end{array}\right) .
$$

It is straightforward to verify that

$$
M=P \Lambda P^{-1}
$$

and

$$
\Lambda=P^{-1} M P \text {. }
$$


Pre-multiply equation (C.9) by $P^{-1}$ to obtain

$$
P^{-1} Y_{x}(x)=P^{-1} M P P^{-1} Y(x)+P^{-1} G(x)
$$

which implies the diagonalized system

$$
W_{x}(x)=\Lambda W(x)+H(x)
$$

where

$$
W(x) \equiv P^{-1} Y(x)
$$

and

$$
H(x) \equiv P^{-1} G(x) .
$$

Therefore,

$$
H(x)=\frac{1}{m^{H}+m^{L}}\left(\begin{array}{c}
-\frac{1}{\phi^{H}} g^{H}(x)+\frac{1}{\phi^{L}} g^{L}(x) \\
-m^{L} \frac{1}{\phi^{H}} g^{H}(x)-m^{H} \frac{1}{\phi^{L}} g^{L}(x)
\end{array}\right) .
$$

The diagonalized system is

$$
\left(\begin{array}{l}
d w_{1}(x) / d x \\
d w_{2}(x) / d x
\end{array}\right)=\left(\begin{array}{cc}
m^{H}+m^{L} & 0 \\
0 & 0
\end{array}\right)\left(\begin{array}{l}
w_{1}(x) \\
w_{2}(x)
\end{array}\right)+\frac{1}{m^{H}+m^{L}}\left(\begin{array}{c}
-\frac{1}{\phi^{H}} g^{H}(x)+\frac{1}{\phi^{L}} g^{L}(x) \\
-m^{L} \frac{1}{\phi^{H}} g^{H}(x)-m^{H} \frac{1}{\phi^{L}} g^{L}(x)
\end{array}\right)
$$

Because the equation for $d w_{i}(x) / d x$ is a first-order linear constant coefficient nonhomogeneous ODE in $w_{i}(x)$, but is independent of $w_{j}(x), j \neq i$, the general solution is

$$
W(x)=\left(\begin{array}{c}
e^{\left(m^{H}+m^{L}\right) x}\left[c_{1}+\frac{1}{m^{H}+m^{L}} \int_{0}^{x} e^{-\left(m^{H}+m^{L}\right) z}\left(-\frac{1}{\phi^{H}} g^{H}(z)+\frac{1}{\phi^{L}} g^{L}(z)\right) d z\right] \\
c_{2}-\frac{1}{m^{H}+m^{L}} \int_{0}^{x}\left(m^{L} \frac{1}{\phi^{H}} g^{H}(z)+m^{H} \frac{1}{\phi^{L}} g^{L}(z)\right) d z
\end{array}\right) .
$$

Note that

$$
W(0)=\left(\begin{array}{l}
w_{1}(0) \\
w_{2}(0)
\end{array}\right)=\left(\begin{array}{l}
c_{1} \\
c_{2}
\end{array}\right) .
$$

Use $Y(x)=P W(x)$ so that

$$
\left(\begin{array}{c}
y^{H}(x) \\
y^{L}(x)
\end{array}\right)=\left(\begin{array}{cc}
m^{H} & 1 \\
-m^{L} & 1
\end{array}\right)\left(\begin{array}{c}
w_{1}(x) \\
w_{2}(x)
\end{array}\right)
$$

which implies

$$
\left(\begin{array}{c}
y^{H}(x) \\
y^{L}(x)
\end{array}\right)=\left(\begin{array}{c}
m^{H} w_{1}(x)+w_{2}(x) \\
-m^{L} w_{1}(x)+w_{2}(x)
\end{array}\right)
$$


and hence

$$
y^{H}(x)-y^{L}(x)=\left(m^{H}+m^{L}\right) w_{1}(x) .
$$

Substitute the boundary condition $y^{L}(0)=0$ from equation (C.7) into equation (C.30) and use $w_{1}(0)=c_{1}$ from equation (C.27) to obtain

$$
y^{H}(0)=\left(m^{H}+m^{L}\right) c_{1} .
$$

To determine the value of the constant $c_{1}$, apply the boundary condition $y_{x}^{H}\left(x^{*}\right)=0$ from equation (C.6). Observe that equation (C.5) implies $y_{x}^{H}(x)=m^{H}\left(y^{H}(x)-y^{L}(x)\right)-$ $\frac{1}{\phi^{H}} g^{H}(x)$ and then use equation (C.30) to obtain

$$
y_{x}^{H}(x)=m^{H}\left(m^{H}+m^{L}\right) w_{1}(x)-\frac{1}{\phi^{H}} g^{H}(x) .
$$

Evaluate equation (C.32) at $x=x^{*}$, set the right hand side of the resulting equation equal to zero and rearrange to obtain

$$
m^{H}\left(m^{H}+m^{L}\right) w_{1}\left(x^{*}\right)=\frac{1}{\phi^{H}} g^{H}\left(x^{*}\right)
$$

Evaluate the first element of the right hand side of equation (C.26) at $x=x^{*}$ and substitute the resulting expression for $w_{1}\left(x^{*}\right)$ into equation (C.33) to obtain

$$
\begin{aligned}
c_{1}= & \frac{1}{m^{H}\left(m^{H}+m^{L}\right)} \frac{1}{\phi^{H}} g^{H}\left(x^{*}\right) e^{-\left(m^{H}+m^{L}\right) x^{*}} \\
& -\frac{1}{m^{H}+m^{L}} \int_{0}^{x^{*}} e^{-\left(m^{H}+m^{L}\right) z}\left(-\frac{1}{\phi^{H}} g^{H}(z)+\frac{1}{\phi^{L}} g^{L}(z)\right) d z .
\end{aligned}
$$

Substitute the value for $c_{1}$ from equation (C.34) into equation (C.31) and use $m^{H} \phi^{H}=\mu^{L}$ to obtain

$$
y^{H}(0)=\frac{1}{\mu^{L}} g^{H}\left(x^{*}\right) e^{-\left(m^{H}+m^{L}\right) x^{*}}-\int_{0}^{x^{*}} e^{-\left(m^{H}+m^{L}\right) z}\left(-\frac{1}{\phi^{H}} g^{H}(z)+\frac{1}{\phi^{L}} g^{L}(z)\right) d z .(
$$

To make following expressions more compact, define

$$
b \equiv-\left(m^{H}+m^{L}\right)=-\left(\frac{\mu^{L}}{\phi^{H}}+\frac{\mu^{H}}{\phi^{L}}\right)>0,
$$

so equation (C.35) can be rewritten as

$$
y^{H}(0)=\frac{1}{\mu^{L}} g^{H}\left(x^{*}\right) e^{b x^{*}}-\int_{0}^{x^{*}} e^{b z}\left(-\frac{1}{\phi^{H}} g^{H}(z)+\frac{1}{\phi^{L}} g^{L}(z)\right) d z .
$$

We are particularly interested in $y^{H}(0)$ in the three cases below, which have different specifications of the pair of indicator functions $g^{H}(x)$ and $g^{L}(x)$. 
Case $1 g^{H, 1}(x)=g^{L, 1}(x)=1$ for $0 \leq x \leq x^{*}$, so that

$$
y^{H, 1}(0)=E_{0}\left\{\int_{0}^{\tau} d t\right\}=E_{0}\{\tau\}>0 .
$$

In this case, the expression for $y^{H}(0)$ in equation (C.37) becomes

$$
y^{H, 1}(0)=\frac{1}{\mu^{L}} e^{b x^{*}}+\left(\frac{1}{\phi^{H}}-\frac{1}{\phi^{L}}\right) \frac{1}{b}\left(e^{b x^{*}}-1\right)>0 .
$$

Case $2 g^{H, 2}(x)=0, g^{L, 2}(x)=1_{\left\{x_{t}<\Delta, s=L\right\}}$ for $0 \leq x \leq x^{*}$, so that

$$
y^{H, 2}(0, \Delta)=E_{0}\left\{\int_{0}^{\tau} 1_{\left\{x_{t}<\Delta, s=L\right\}} d t\right\}=F(\Delta, L) E_{0}\{\tau\}>0 .
$$

In this case, the expression for $y^{H}(0)$ in equation (C.37) becomes

$$
y^{H, 2}(0, \Delta)=-\frac{1}{\phi^{L}} \frac{1}{b}\left(e^{b \Delta}-1\right) .
$$

Case $3 g^{H, 3}(x)=1_{\left\{x_{t}<\Delta, s=H\right\}}, g^{L, 3}(x)=0$ for $0 \leq x \leq x^{*}$, so that

$$
y^{H, 3}(0, \Delta)=E_{0}\left\{\int_{0}^{\tau} 1_{\left\{x_{t}<\Delta, s=H\right\}} d t\right\}=F(\Delta, H) E_{0}\{\tau\}>0 .
$$

In this case, $g^{H, 3}(x)=1$ for $0 \leq x<\Delta \leq x^{*}$, but $g^{H, 3}\left(x^{*}\right)=0$, so the expression for $y^{H}(0)$ in equation (C.37) becomes

$$
y^{H, 3}(0, \Delta)=\frac{1}{\phi^{H}} \frac{1}{b}\left(e^{b \Delta}-1\right)>0 .
$$

Equations (C.38) and (C.40) imply that $F(\Delta, L)=\frac{y^{H, 2}(0, \Delta)}{y^{H, 1}(0)}$ so that equations (C.39) and (C.41) imply

$$
F(\Delta, L)=\frac{-1}{\phi^{L}} \frac{1}{\frac{b}{\mu^{L}} e^{b x^{*}}+\left(\frac{1}{\phi^{H}}-\frac{1}{\phi^{L}}\right)\left(e^{b x^{*}}-1\right)}\left(e^{b \Delta}-1\right)>0, \text { for } 0 \leq \Delta \leq x^{*} .
$$

Similarly, equations (C.38) and (C.42) imply that $F(\Delta, H)=\frac{y^{H, 3}(0, \Delta)}{y^{H, 1}(0)}$ so that equations (C.39) and (C.43) imply

$$
F(\Delta, H)=\frac{1}{\phi^{H}} \frac{1}{\frac{b}{\mu^{L}} e^{b x^{*}}+\left(\frac{1}{\phi^{H}}-\frac{1}{\phi^{L}}\right)\left(e^{b x^{*}}-1\right)}\left(e^{b \Delta}-1\right)>0, \text { for } 0 \leq \Delta<x^{*} .
$$

Finally, to express the distribution even more compactly, define

$$
a \equiv \frac{1}{\frac{b}{\mu^{L}} e^{b x^{*}}+\left(\frac{1}{\phi^{H}}-\frac{1}{\phi^{L}}\right)\left(e^{b x^{*}}-1\right)}>0
$$


and use the definition of $a$ to rewrite equations (C.44) and (C.45) as

$$
\begin{aligned}
& F(x, L)=\frac{-1}{\phi^{L}} a\left(e^{b x}-1\right)>0, \text { for } 0 \leq x \leq x^{*} \\
& F(x, H)=\frac{1}{\phi^{H}} a\left(e^{b x}-1\right)>0, \text { for } 0 \leq x \leq x^{*}
\end{aligned}
$$

and hence

$$
1-F\left(x^{*}, L\right)-F\left(x^{*}, H\right)=\frac{1}{\mu^{L}} a b e^{b x^{*}}>0 .
$$

Finally, equations (C.47) and (C.48) can be represented as

$$
F(x, j)=\frac{1}{\left|\phi^{(j)}\right|} a\left(e^{b x}-1\right)>0, \text { for } j \in\{H, L\} \text { and } 0 \leq x \leq x^{*}
$$

\section{Implementing the Two-Stage Procedure}

This appendix provides additional details for the two-stage procedure. Stage 1 of the 2stage procedure is simply an OLS regression within Regime $L$. Stage 2 uses the distance $\Theta$ between points $\mathrm{A}$ and $\mathrm{D}$. The coordinates of point $\mathrm{A}$ are $\left(q_{A}, q_{M}\right)=\left(q_{A}^{H}\left(x^{*}\right), q_{A}^{H}\left(x^{*}\right)\right)$ because $q_{M}^{H}\left(x^{*}\right)=q_{A}^{H}\left(x^{*}\right)$. Point $\mathrm{D}$ is the point on the fitted regression line from Stage 1 , $\beta_{0}+\beta_{q}^{I I I} q_{A}$, that is vertically below Point $\mathrm{A}$, where $q_{A}=q_{A}^{H}\left(x^{*}\right)$. Therefore, the coordinates of Point $\mathrm{D}$ are $\left(q_{A}, q_{M}\right)=\left(q_{A}^{H}\left(x^{*}\right), \beta_{0}+\beta_{q}^{I I I} q_{A}^{H}\left(x^{*}\right)\right)$, so $\Theta$, the vertical distance between Points $\mathrm{A}$ and $\mathrm{D}$, is

$$
\Theta=\left(1-\beta_{q}^{I I I}\right) q_{A}^{H}\left(x^{*}\right)-\beta_{0}
$$

Use the definition of the approximation error $\zeta$ in equation (36) to eliminate $\beta_{0}$ from equation (D.1) and obtain

$$
\Theta=\left(1-\beta_{q}^{I I I}\right) q_{A}^{H}\left(x^{*}\right)+\zeta-q_{M}^{L}\left(x^{*}\right)+\beta_{q}^{I I I} q_{A}^{L}\left(x^{*}\right) .
$$

Next use the definitions $q_{A}^{j}(x) \equiv v^{j}(x)-x$ and $q_{M}^{L}(x) \equiv \frac{v^{L}(x)}{v_{x}^{L}(x)}-x$ in equation (D.2) to obtain

$$
\Theta=\left(1-\beta_{q}^{I I I}\right) v^{H}\left(x^{*}\right)+\zeta-\frac{v^{L}\left(x^{*}\right)}{v_{x}^{L}\left(x^{*}\right)}+\beta_{q}^{I I I} v^{L}\left(x^{*}\right) .
$$

Use the facts from equation (27) and Proposition 3 that $v^{H}\left(x^{*}\right)=\frac{\Phi^{H}+\Phi^{L}}{1-\Gamma}, v_{x}^{L}\left(x^{*}\right)=\frac{\rho+\mu^{L}}{\mu^{L}}$, and $v^{L}\left(x^{*}\right)=v_{x}^{L}\left(x^{*}\right) \frac{\Phi^{L}+\Gamma \Phi^{H}}{1-\Gamma}$ to rearrange equation (D.3) to obtain an expression for $\Theta$ in 
terms of the parameters of the firm's problem and the estimated values of $\beta_{q}^{I I I}$ and the approximation error $\zeta$,

$$
\Theta=\left(1-\beta_{q}^{I I I}\right) \Phi^{H}+\beta_{q}^{I I I} \frac{\rho}{\mu^{L}} \frac{\Phi^{L}+\Gamma \Phi^{H}}{1-\Gamma}+\zeta .
$$

Remarkably, the expression for $\Theta$ in equation (D.4) does not include $x^{*}$, so $\Theta$ is easily calculated from the fundamental parameters and the results of the univariate regression in Stage 1, without having to compute $x^{*}$.

Use $q_{A}^{H}\left(x^{*}\right)-q_{A}^{L}\left(x^{*}\right)=v^{H}\left(x^{*}\right)-v^{L}\left(x^{*}\right)$ and the expression for the distance $\Theta$ shown in equation (D.3), along with Term 2 and Term 3 from equation (40), to obtain

$$
\text { Term } 2+\operatorname{Term} 3=\frac{1}{\theta}(\Theta-\zeta)=\frac{1}{\theta}\left(\phi^{H}-\phi^{L}\right) \widehat{\beta}_{\phi}-\frac{1}{\theta} \zeta .
$$

After the second equals sign in equation (D.5), the first term is the regression impact of cash flow in equation (39) and the second term is proportional to the approximation error $\zeta$. 